\title{
MEASUREMENTS OF THE SUNYAEV-ZEL'DOVICH EFFECT IN MACS J0647.7+7015 AND MACS J1206.2-0847 AT HIGH ANGULAR RESOLUTION WITH MUSTANG
}

\author{
Alexander H. Young ${ }^{1,2,16}$, Tony Mroczkowski ${ }^{3,17}$, Charles Romero ${ }^{4,5}$, Jack Sayers $^{6}$, Italo Balestra ${ }^{7}$, Tracy E. Clarke ${ }^{8}$, \\ ${\text { Nicole } \text { Czakon }^{9} \text {, Mark Devlin }}^{1}$, Simon R. Dicker ${ }^{1}$, Chiara Ferrari ${ }^{10}$, Marisa Girardi ${ }^{7,11}$, Sunil Golwala ${ }^{6}$, \\ Huib Intema $^{12}$, Phillip M. Korngut ${ }^{6}$, Brian S. Mason ${ }^{4}$, Amata Mercurio ${ }^{13}$, Mario Nonino ${ }^{7}$, Erik D. Reese ${ }^{14}$, \\ Piero Rosati ${ }^{15}$, Craig Sarazin ${ }^{5}$, and Keilchi Umetsu ${ }^{9}$ \\ ${ }^{1}$ Department of Physics and Astronomy, University of Pennsylvania, 209 South 33rd Street, Philadelphia, PA 19104, USA; alyoung@ sas.upenn.edu \\ ${ }^{2}$ NASA Goddard Space Flight Center, 8800 Greenbelt Rd, Greenbelt, MD 20771, USA \\ ${ }^{3}$ U.S. Naval Research Laboratory, 4555 Overlook Ave SW, Washington, DC 20375, USA \\ ${ }^{4}$ National Radio Astronomy Observatory, 520 Edgemont Rd. Charlottesville, VA 22903, USA \\ ${ }^{5}$ Department of Astronomy, University of Virginia, P.O. Box 400325, Charlottesville, VA 22901, USA \\ ${ }^{6}$ Department of Physics, Math, and Astronomy, California Institute of Technology, 1200 East California Blvd, Pasadena, CA 91125, USA \\ ${ }_{7}^{7}$ INAF-Osservatorio Astronomico di Trieste, Via G. B. Tiepolo 11, I-34143 Trieste, Italy \\ ${ }^{8}$ U.S. Naval Research Laboratory, 4555 Overlook Ave SW, Washington, DC 20375, USA \\ ${ }^{9}$ Institute of Astronomy and Astrophysics, Academia Sinica, P.O. Box 23-141, Taipei 10617, Taiwan \\ ${ }^{10}$ Laboratoire Lagrange, UMR 7293, Université de Nice Sophia-Antipolis, CNRS, Observatoire de la Côte d'Azur, F-06300 Nice, France \\ ${ }^{11}$ Dipartimento di Fisica Dell'Universitá degli Studi di Trieste-Sezione di Astronomia, Via Tiepolo 11, I-34143 Trieste, Italy \\ ${ }^{12}$ National Radio Astronomy Observatory, P.O. Box O, 1003 Lopezville Road, Socorro, NM 87801-0387, USA \\ 13 INAF-Osservatorio Astronomico di Capodimonte, Via Moiariello 16, I-80131 Napoli, Italy
${ }^{14}$ Department of Physics, Astronomy, and Engineering, Moorpark College, 7075 Campus Rd., Moorpark, CA 93021, USA \\ ${ }^{15}$ Dipartimento di Fisica e Scienze della Terra, Universitá di Ferrara, Via Saragat, 1, I-44122, Ferrara, Italy \\ Received 2014 November 1; accepted 2015 July 20; published 2015 August 21
}

\begin{abstract}
We present high resolution $\left(9^{\prime \prime}\right)$ imaging of the Sunyaev-Zel'dovich Effect (SZE) toward two massive galaxy clusters, MACS J0647.7+7015 $(z=0.591)$ and MACS J1206.2-0847 $(z=0.439)$. We compare these $90 \mathrm{GHz}$ measurements, taken with the Multiplexed Squid/TES Array at Ninety Gigahertz (MUSTANG ) receiver on the Green Bank Telescope, with generalized Navarro-Frenk-White (gNFW) models derived from Bolocam $140 \mathrm{GHz}$ SZE data as well as maps of the thermal gas derived from Chandra X-ray observations. We adopt a serial-fitting approach, in which gNFW models are first fit to the Bolocam data and then compared to the MUSTANG data to determine an overall best-fit model. For MACS J0647.7+7015, we find a gNFW profile with core slope parameter $\gamma=0.9$ fits the MUSTANG image with $\chi_{\text {red }}^{2}=1.005$ and probability to exceed (PTE) $=0.34$. For MACS J1206.2-0847, we find $\gamma=0.7, \chi_{\text {red }}^{2}=0.993$, and PTE $=0.70$. In addition, we find a significant $(>3 \sigma)$ residual SZE feature in MACS J1206.2-0847 coincident with a group of galaxies identified in Very Large Telescope data and filamentary structure found in a weak-lensing mass reconstruction. We suggest the detected sub-structure may be the SZE decrement from a low mass foreground group or an infalling group. Giant Metrewave Radio Telescope measurements at $610 \mathrm{MHz}$ reveal diffuse extended radio emission to the west, which we posit is either an active galactic nucleus-driven radio lobe, a bubble expanding away from disturbed gas associated with the SZE signal, or a bubble detached and perhaps re-accelerated by sloshing within the cluster. Using the spectroscopic redshifts available, we find evidence for a foreground $(z=0.423)$ or infalling group, coincident with the residual SZE feature.
\end{abstract}

Key words: cosmic background radiation - cosmology: observations galaxies: clusters: individual (MACS J0647.7+7015, MACS J1206.2-0847) galaxies: clusters: intracluster medium - X-rays: galaxies: clusters

\section{INTRODUCTION}

Clusters of galaxies are the largest gravitationally bound systems in the universe and encompass volumes great enough to be considered representative samples of the universe at large. By mass, clusters comprise dark matter $(\sim 85 \%)$, hot plasma known as the intracluster medium (ICM; $\sim 12 \%$ ), and a few percent stars and galaxies.

The diverse matter content of clusters provides a wide range of observables across the electromagnetic spectrum. X-ray and millimeter-wave Sunyaev-Zel'dovich Effect (SZE) observations measure the thermodynamic properties of the ICM, such as density and temperature, which provides insight into the

\footnotetext{
16 NASA Postdoctoral Fellow.

17 National Research Council Fellow.
}

formation history and evolution of the cluster as well as its current dynamical state. Radio observations have discovered diffuse synchrotron emission in many galaxy clusters, typically associated with merger-induced shock fronts, turbulence, or active galactic nucleus (AGN) activity (e.g., van Weeren et al. 2011; Cassano et al. 2012). Optical observations reveal the individual galaxy population and, through dynamical and lensing studies, allow us to infer the cluster mass distribution.

The SZE is a distortion of the cosmic microwave background (CMB) caused by inverse Compton scattering of photons off the electrons of the hot ICM trapped in the gravitational potential well of clusters. The SZE is directly proportional to the electron pressure of the ICM integrated along the line of sight (Sunyaev and Zel'dovich 1972). Measurements of the SZE on small spatial scales in galaxy 
Table 1

Cluster Properties

\begin{tabular}{lccccr}
\hline \hline Cluster & $z$ & $\begin{array}{c}R_{500} \\
(\mathrm{Mpc})\end{array}$ & $\begin{array}{c}P_{500} \\
\left(10^{-3} \mathrm{keV} \mathrm{cm}^{-3}\right)\end{array}$ & $\begin{array}{c}M_{500} \\
\left(10^{14} M_{\odot}\right)\end{array}$ & $\begin{array}{c}k_{\mathrm{B}} T_{\mathrm{X}} \\
(\mathrm{keV})\end{array}$ \\
\hline MACS J0647.7 & 0.591 & $1.26 \pm 0.06$ & $9.23 \pm 2.57$ & $10.9 \pm 1.6$ & $1.5 \pm 1.1$ \\
MACS J1206.2 & 0.439 & $1.61 \pm 0.08$ & $10.59 \pm 3.07$ & $19.2 \pm 3.0$ & $1.5 \pm 1.3$ \\
\hline
\end{tabular}

Notes. X-ray-derived cluster properties, reproduced from Mantz et al. (2010).

${ }^{a}$ Values of $Y_{500}$ are derived from the values reported in Mantz et al. (2010) using Equation (6).

clusters provide a powerful probe of astrophysical phenomena (e.g., Kitayama et al. 2004; Korngut et al. 2011). For reviews of the SZE and its scientific applications, see Birkinshaw (1999) and Carlstrom et al. (2002).

In this work, we present high-resolution SZE measurements of two galaxy clusters, MACS J0647.7+7015 and MACS J1206.2-0847, taken with the Multiplexed Squid/ TES Array at Ninety Gigahertz (MUSTANG). We carry out a multi-wavelength investigation using the comprehensive data sets provided by the Cluster Lensing and Supernova survey with Hubble (CLASH) program (Postman et al. 2012). The X-ray measured properties of MACS J0647.7+7015 (MACS J0647.7) and MACS J1206.2-0847(MACS J1206.8) from Mantz et al. (2010) are summarized in Table 1.

The organization of this paper is as follows. In Section 2 we discuss the MUSTANG, X-ray, and Bolocam observations and data reduction. In Sections 3 and 4, we discuss the ICM modeling and least-squares fitting procedure used in the analysis of the MUSTANG and Bolocam data. The results are discussed and summarized in Section 5. Throughout this paper, we adopt a flat, $\Lambda$-dominated cosmology with $\Omega_{M}=0.3$, $\Omega_{\Lambda}=0.7$, and $H_{0}=70 \mathrm{~km} \mathrm{~s}^{-1} \mathrm{Mpc}^{-1}$ consistent with Planck results (Planck Collaboration et al. 2014). At the redshifts of MACS J0647.7+7015 $(z=0.591)$ and MACS J1206.2-0847 $(z=0.439), 1^{\prime \prime}$ corresponds to 6.64 and $5.68 \mathrm{kpc}$, respectively.

\section{OBSERVATIONS AND DATA REDUCTION}

\subsection{The CLASH Sample}

In this paper, we present MUSTANG observations of MACS J0647.7+7015 and MACS J1206.2-0847. Basic characteristics of these clusters are summarized in Tables 1 and 2, as part of an ongoing program to provide high-resolution SZE images of the CLASH clusters accessible from MUSTANG's location on the Green Bank Telescope (GBT; Jewell \& Prestage 2004). The 25 clusters in CLASH have comprehensive multi-wavelength coverage, including deep 16-band HST optical imaging, relatively low resolution SZE measurements, and X-ray observations with Chandra and XMM-Newton. These clusters are generally dynamically relaxed, span redshifts from $0.2 \lesssim z \lesssim 0.9$, and masses from $3 \times 10^{14} \lesssim M_{500} / M_{\odot}$ $\lesssim 2 \times 10^{15}$. For a comprehensive description of the CLASH sample and selection criteria see Postman et al. (2012).

\subsection{MUSTANG}

MUSTANG is a 64-pixel array of Transition Edge Sensor (TES) bolometers spaced at $0.6 f \lambda$ operating at $90 \mathrm{GHz}$ on the $100 \mathrm{~m}$ GBT. MUSTANG has an instantaneous field of view (FOV) of $42^{\prime \prime}$ and angular resolution of $9^{\prime \prime}$. For more information about MUSTANG, refer to Dicker et al. (2008).

MUSTANG has measured the SZE at high resolution in several galaxy clusters to date, including RX J1347.5-1145,
Table 2

MUSTANG Observation Overview

\begin{tabular}{lcccc}
\hline \hline \multirow{2}{*}{ Cluster } & \multicolumn{2}{c}{ Centroid (J2000) } & \multirow{2}{*}{ Obs. Time } & \multirow{2}{*}{ Peak $|\mathrm{S} / \mathrm{N}|$} \\
\cline { 2 - 3 } & R.A. & Decl. & $(\mathrm{hr})$ & \\
\hline MACS J0647.7 & $06: 47: 50.5$ & $+70: 14: 53$ & 16.4 & 8.1 \\
MACS J1206.2 & $12: 06: 12.5$ & $-08: 48: 07$ & 12.1 & 4.1 \\
\hline
\end{tabular}

Note. MUSTANG observations were carried out between 2011 February and 2013 January.

CL J1226.9+3332, MACS J0717.5+3745, MACS J0744.9 +3927 , and Abell 1835. MUSTANG observations confirmed $(>13 \sigma)$ the presence of merger activity in RX J1347.5-1145 (Mason et al. 2010; Ferrari et al. 2011; Korngut et al. 2011) that was hinted at by observations with the Nobeyama $45 \mathrm{~m}$ telescope (Komatsu et al. 2001) and the $30 \mathrm{~m}$ IRAM telescope (Pointecouteau et al. 1999). Korngut et al. (2011) used MUSTANG data to discover a shock in MACS J0744.9 +3927 that was previously undetected. In MACS J0717.5 +3745 , Mroczkowski et al. (2012) used MUSTANG data to report a pressure enhancement due to shock-heated gas immediately adjacent to extended radio emission.

The MUSTANG observations and data reduction in this work largely follow the procedure described in Mason et al. (2010) and Korngut et al. (2011). We direct the telescope in a Lissajous daisy scan pattern with seven pointing centers surrounding the cluster core. This mosaic provides deep, uniform coverage in the cluster core and falls off steeply beyond a radius of $\sim 30^{\prime \prime}$.

During observations, nearby bright compact radio sources were mapped once every 30 minutes to track changes in the beam profile including drifts in telescope gain and pointing offsets. Typically, if there was a substantial $(\sim 20 \%)$ drop in the peak of the beam profile, or if the beam width exceeded $10^{\prime \prime}$, we re-derived the GBT active surface corrections using an outof-focus holography technique (Nikolic et al. 2007). We used the blazar JVAS $0721+7120$ for MACS J0647.7+7015 and the quasar JVAS 1229+0203 for MACS J1206.2-0847 to determine these gains and focusing corrections. Planets or stable quasars including Mars, Saturn, and 3C286 (Agudo et al. 2012) were mapped at least once per observation session to provide absolute flux calibration. Fluxes for planets were calculated based on brightness temperatures from WMAP observations (Weiland et al. 2011). The absolute flux of the data is calibrated to an accuracy of $10 \%$. Throughout this work, we ignore the systematic uncertainty from the absolute flux calibration and quote only the statistical uncertainties.

The MUSTANG data are reduced using a custom IDL pipeline. The bolometric timestreams are high-pass filtered by subtracting a high order Legendre polynomial determined by the scan speed of the telescope. For a typical 300 s scan, and 
$40^{\prime \prime} \mathrm{s}^{-1}$ scan speed, we choose a $\sim 100$ th-order Legendre polynomial, corresponding to a cutoff frequency of $\sim 0.3 \mathrm{~Hz}$. In order to remove atmospheric noise on large angular scales, we subtract the mean measurement from all detectors for each sample in time. This also removes astronomical signals on angular scales larger than the FOV of the instrument $\left(\approx 42^{\prime \prime}\right)$.

The standard deviation, $\sigma$, of each individual detector timestream is computed, and a corresponding weight, $w$, is determined according to $w=1 / \sigma^{2}$. To produce a "signal map," the timestreams are binned into $1^{\prime \prime} \times 1^{\prime \prime}$ spatial pixels and smoothed with the MUSTANG point-spread function (PSF), or beam. We compute the weight for each pixel of the smoothed data map to produce a "weight map." We multiply the signal map by the square root of the weight map to generate a map in units of signal-to-noise ratio $(\mathrm{S} / \mathrm{N})$ - the "SNR map."

We generate an independent "noise map" by flipping the sign of measurements from every other scan and binning the data into a grid with the same pixel size as the signal map. As we do for the signal map, we use the pixel weights to convert the noise map to units of $\mathrm{S} / \mathrm{N}$, referred to as a "noise SNR map." We define a scale factor, $\sigma_{\mathrm{N}}$, as the standard deviation of the noise SNR map. For an ideal noise distribution, $\sigma_{\mathrm{N}}=1$. We can therefore use $\sigma_{\mathrm{N}}$ as a normalization factor to account for "non-ideal" noise features, such as correlations between detectors. Typically, we find $\sigma_{\mathrm{N}} \approx 1.5$, which means that the timestream-based weight maps are under-estimating the noise.

\subsection{Bolocam}

Bolocam is a 144-pixel bolometer array at the Caltech Submillimeter Observatory capable of operating at 140 and $268 \mathrm{GHz}$, with resolutions of $31^{\prime \prime}$ and $58^{\prime \prime}$, respectively, and an instantaneous FOV $8^{\prime}$ in diameter. For more details on the Bolocam instrument see Haig et al. (2004).

As part of a larger cluster program (Sayers et al. 2013; Czakon et al. 2015), Bolocam was used to obtain high significance SZE images of MACS J0647.7+7015 $(\mathrm{S} / \mathrm{N}=14.4)$ and MACS J1206.2-0847 $(\mathrm{S} / \mathrm{N}=21.7)$. In this work, we make use of these Bolocam data to constrain bulk models of the SZE emission based on generalized NavarroFrenk-White (gNFW) pressure profiles (Nagai et al. 2007), including the specific case of the "universal pressure profile" (Arnaud et al. 2010, hereafter A10). The model fitting procedure is described in Section 4.2, and the details of the Bolocam data, along with its reduction are given in Sayers et al. (2013, hereafter S13) and Czakon et al. (2015).

\subsection{Chandra}

Archival Chandra X-ray data were reduced using CIAO ${ }^{18}$ version 4.5 with calibration database version 4.5.5. MACS J0647.7+7015 was observed for a total exposure time of $39 \mathrm{ks}$ (ObsIDs 3196 and 3584). MACS J1206.2-0847 was observed for $24 \mathrm{ks}$ (ObsID 3277). For details on the X-ray data processing see Reese et al. (2010).

\section{ICM ANALYSIS}

The thermal SZE intensity is described by

$$
\frac{\Delta I_{\mathrm{SZE}}}{I_{0}}=g\left(\nu, T_{\mathrm{e}}\right) y,
$$

\footnotetext{
${ }^{18}$ http://cxc.harvard.edu/ciao/
}

where $\nu$ is the observed frequency, $T_{\mathrm{e}}$ is the electron temperature, $y$ is the Compton- $y$ parameter (described below), and the primary $\mathrm{CMB}$ surface brightness is $I_{0}=2\left(k_{\mathrm{B}} T_{\mathrm{CMB}}\right)^{3}(h c)^{-2}=2.7033 \times 10^{8} \mathrm{Jy} \mathrm{sr}^{-1}$. The function $g\left(\nu, T_{\mathrm{e}}\right)$ describes the frequency dependence of the thermal SZE (Carlstrom et al. 2002) and includes the relativistic corrections of Itoh et al. (1998) and Itoh \& Nozawa (2004). At $90 \mathrm{GHz}$, the SZE manifests as a decrement in the CMB intensity.

The frequency-independent Compton-y parameter is defined as

$$
y \equiv \frac{\sigma_{\mathrm{T}}}{m_{\mathrm{e}} c^{2}} \int n_{\mathrm{e}} k_{\mathrm{B}} T_{\mathrm{e}} d \ell
$$

where $\sigma_{\mathrm{T}}$ is the Thomson cross-section, $m_{\mathrm{e}} c^{2}$ is the electron rest energy, and the integration is along the line of sight $\ell$. Therefore, by the ideal gas law, the SZE intensity is proportional to the ICM electron pressure $P_{\mathrm{e}}=n_{\mathrm{e}} k_{\mathrm{B}} T_{\mathrm{e}}$ integrated along the line of sight. The total SZE signal, integrated within an aperture $\theta=R / D_{A}$, is often expressed in units of solid angle, where $Y_{\mathrm{SZ}}[\mathrm{sr}]=\int y d \Omega$ or in distance units where $Y\left[\mathrm{Mpc}^{2}\right]=Y_{\mathrm{SZ}} D_{A}^{2}$.

The X-ray surface brightness (in units of ergs $\mathrm{cm}^{-2} \mathrm{~s}^{-1}$ ) is

$$
S_{\mathrm{X}}=\frac{1}{4 \pi(1+z)^{4}} \int n_{\mathrm{e}}^{2} \Lambda_{\mathrm{ee}}\left(T_{\mathrm{e}}, Z\right) d \ell
$$

where $\Lambda_{\mathrm{ee}}\left(T_{\mathrm{e}}, Z\right)$ is the $\mathrm{X}$-ray cooling function (in units of ergs $\mathrm{cm}^{3} \mathrm{~s}^{-1}$ ), and $Z$ is the abundance of heavy elements relative to that in the Sun. Assuming the temperature is constant along the line of sight,

$$
n_{\mathrm{e}} \approx \sqrt{\frac{4 \pi(1+z)^{3} S_{\mathrm{X}}}{\Lambda_{\mathrm{ee}}\left(T_{\mathrm{e}}, Z\right) \ell}} .
$$

We approximate Equation (2) as $y \approx \sigma_{\mathrm{T}} /\left(m_{\mathrm{e}} c^{2}\right) n_{\mathrm{e}} k_{\mathrm{B}} T_{\mathrm{e}} \ell$ and use Equation (3) to derive from the X-ray data a "pseudo"-y value, ${ }^{19}$ given by

$$
y=\frac{\sigma_{\mathrm{T}} k_{\mathrm{B}} T_{\mathrm{e}}}{m_{\mathrm{e}} c^{2}} \sqrt{\frac{4 \pi(1+z)^{3} S_{\mathrm{X}} \ell}{\Lambda_{\mathrm{ee}}\left(T_{\mathrm{e}}, Z\right)}} .
$$

We assume a single value for $\ell$ across the map. This "slab approximation" is not completely valid, but has been used in other works for qualitative comparisons to Compton-y maps (see Mroczkowski et al. 2012; Planck Collaboration et al. 2013b). It assumes that the cluster temperature is constant along the line of sight and that the average ratio of Compton- $y$ to $\sqrt{S_{\mathrm{X}}}$ is approximately constant azimuthally.

We use a measurement of the integrated Compton- $y\left(Y_{\mathrm{SZ}} D_{A}^{2}\right)$ within $R<1^{\prime}$ from Bolocam to infer $\ell$ and normalize the X-ray pseudo-y map accordingly.

The X-ray data for MACS J0647.7+7015 and MACS J1206.2-0847 were not deep enough for detailed temperature mapping. We fit the X-ray spectra in large spatial bins and found best-fit temperatures consistent with Mantz et al. (2010). For simplicity, we assume an isothermal distribution with the the $k_{\mathrm{B}} T_{\mathrm{e}}$ values determined by Mantz et al. (2010). We note that for each of these clusters, the assumption of an isothermal

\footnotetext{
19 The "pseudo" distinction is used because $\ell$ is not constrained by the X-ray data alone.
} 
Table 3

gNFW Model Parameters

\begin{tabular}{lccccc}
\hline \hline Model & $P_{0}$ & $C_{500}$ & $\gamma$ & $\alpha$ & $\beta$ \\
\hline S13 ensemble & 4.29 & 1.18 & 0.67 & 0.86 & 3.67 \\
S13 cool-core & 0.65 & 1.18 & 1.37 & 2.79 & 3.51 \\
S13 disturbed & 17.3 & 1.18 & 0.02 & 0.90 & 5.22 \\
A10 ensemble & 8.40 & 1.18 & 0.31 & 1.05 & 5.49 \\
A10 cool-core & 3.25 & 1.13 & 0.77 & 1.22 & 5.49 \\
A10 disturbed & 3.20 & 1.08 & 0.38 & 1.41 & 5.49 \\
MACS J0647.7 & 0.54 & 0.29 & 0.90 & 1.05 & 5.49 \\
MACS J1206.2 & 1.13 & 0.41 & 0.70 & 1.05 & 5.49 \\
\hline
\end{tabular}

Notes. Best-fit gNFW models from S13, A10, and the best-fit Bolo + MUSTANG models presented in Section 5. The $C_{500}\left(C_{500}=R_{500} / R_{\mathrm{s}}\right)$ values for the Bolo+MUSTANG models are derived from the best-fit scale radius $R_{\mathrm{s}}$ from this work and $R_{500}$ from Mantz et al. (2010).

distribution within $r \lesssim 120^{\prime \prime}$ is reasonable based on the relatively flat radial temperature profiles given in the Archive of Chandra Cluster Entropy Profile Tables (ACCEPT) database (Cavagnolo et al. 2009).

Several measurements have shown that the pressure of the ICM is well described by a gNFW pressure profile (e.g., Nagai et al. 2007; Mroczkowski et al. 2009; A10; Plagge et al. 2010; Planck Collaboration et al. 2013a; S13). In this model, the pressure (in units of $P_{500}$ ) is

$$
\tilde{P}(R)=\frac{P_{0}}{\left(R_{\mathrm{s}}^{-1} R\right)^{\gamma}\left[1+\left(R_{\mathrm{s}}^{-1} R\right)^{\alpha}\right]^{(\beta-\gamma) / \alpha}},
$$

where $R_{\mathrm{s}}$ is the scale radius of the cluster, often given in terms of the concentration parameter $C_{500}\left(R_{\mathrm{s}}=R_{500} / C_{500}\right),{ }^{20} P_{0}$ is the normalization factor, $\gamma$ is the inner slope $\left(R \ll R_{\mathrm{S}}\right), \alpha$ is the intermediate slope $\left(R \sim R_{\mathrm{S}}\right)$, and $\beta$ is the outer slope $\left(R \gg R_{\mathrm{S}}\right)$. $P_{500}$ is defined in Equation (7).

In this work, we focus on the gNFW fit results from A10 and S13. The gNFW model parameters for the respective ensemble samples, in addition to subsets defined according to cluster morphology, are given in Table 3 . We also include the best-fit parameters for MACS J0647.7+7015 and MACS J1206.2-0847 determined in Section 5. Pressure profiles for each of these models, scaled based on the values of $P_{500}, R_{500}$, and $z$ given in Table 1 for each cluster, are shown in Figure 1. We also include plots of the spherically integrated Compton- $y$, $Y_{\text {sph }}(<R)$, given by

$$
Y_{\mathrm{sph}}(<R)=\frac{4 \pi \sigma_{\mathrm{T}}}{m_{\mathrm{e}} c^{2}} \int_{0}^{R} P(r) r^{2} d r .
$$

Here, the volume integrated Compton- $y$ is calculated from the gNFW model rather than from the X-ray derived pseudo- $y$ map. Integrating the pseudo-y map would require an assumed geometry (i.e., spherical symmetry) and would rely on the normalization derived from the Bolocam SZE maps. Therefore, calculating $Y_{\mathrm{sph}}$ from the gNFW model is a more direct approach for the scope of this work.

\footnotetext{
${ }^{20} R_{\Delta}$ is defined as the radius at which the mean interior mass density of a cluster is $\Delta$ times the critical density of the universe at the redshift of the cluster: $M_{\Delta}=(4 \pi / 3) \Delta \rho_{c} R_{\Delta}^{3}$.
}

As in A10, we express $Y_{\text {sph }}$ in units of $Y_{500}$, where

$$
Y_{500}=\frac{\sigma_{\mathrm{T}}}{m_{\mathrm{e}} c^{2}} \frac{4 \pi}{3} R_{500}^{3} P_{500},
$$

and

$$
P_{500}=\left(3.68 \times 10^{-3} \mathrm{keV} \mathrm{cm}^{-3}\right)\left(\frac{M_{500}}{10^{15} M_{\odot}}\right)^{2 / 3} E(z)^{8 / 3},
$$

where $\quad E^{2}(z)=\Omega_{M}(1+z)^{3}+\Omega_{\Lambda} \quad$ (see Nagai et al. 2007; A10; S13). The values of $Y_{500}$ and $P_{500}$ from Equations (6) and (7), respectively, are derived from the cluster properties reported in Mantz et al. (2010) and summarized in Table 1.

\section{MODEL FITTING}

While MUSTANG provides high-resolution imaging, the angular transfer function falls off steeply beyond the instrument FOV $\left(\approx 42^{\prime \prime}=255 \mathrm{kpc}\right.$ at $\left.z=0.5\right)$. Bolocam has a lower resolution, but a larger FOV and therefore is sensitive to the bulk SZE signal on larger angular scales (beyond $\sim 10^{\prime}$ ). A combined Bolocam+MUSTANG model-fitting approach allows us to place better constraints on the ICM characteristics over the full range of angular scales probed by both instruments. In this work, we present the first steps toward a robust joint-fitting procedure. We note that this fitting procedure does not perform a true simultaneous fit to both the MUSTANG and the Bolocam data, which is presented in Romero et al. (2015).

\subsection{Fitting Algorithm}

We begin by constructing a model map, such as a projected gNFW model plus a point source, in units of $\mathrm{Jy} \mathrm{beam}^{-1}$ smoothed to MUSTANG resolution. We simulate an observation of the model by injecting noise from real observations (i.e., the noise maps described in Section 2.2) and then processing the mock observation through the MUSTANG mapmaking pipeline. By subtracting the injected noise from the output map we obtain a filtered model map without residual noise. Examples of these post-processed model maps are presented in Section 5.

To fit the filtered model maps to the data in the map domain we use the general linear least squares fitting approach from Numerical Recipes (Press et al. 1992), outlined briefly below.

We construct an $N \times M$ design matrix $\boldsymbol{A}$, where each element $A_{i j}$ corresponds to a model component (e.g., a point source or gNFW model) $X_{j}$ evaluated at map pixel $x_{i}$. In this work, we allow a single free parameter for each model component, a scalar amplitude, $a_{j}$. We call the $M$-length vector of amplitudes $\boldsymbol{a}$ and define a model vector,

$$
\boldsymbol{d}_{\text {mod }}=\boldsymbol{A} \boldsymbol{a} \text {. }
$$

The goodness of fit statistic, $\chi^{2}$, is given by

$$
\chi^{2}=\left(\boldsymbol{d}-\boldsymbol{d}_{\mathrm{mod}}\right)^{T} \boldsymbol{N}^{-1}\left(\boldsymbol{d}-\boldsymbol{d}_{\mathrm{mod}}\right),
$$

where $\boldsymbol{d}$ represents the measured values of each map pixel and $\boldsymbol{N}$ is the noise covariance matrix, where

$$
N_{i j}=\left\langle n_{i} n_{j}\right\rangle-\left\langle n_{i}\right\rangle\left\langle n_{j}\right\rangle .
$$

Here, $\boldsymbol{n}$ is taken to be pixel values of a noise map, and the covariance matrix is calculated using the ensemble average 

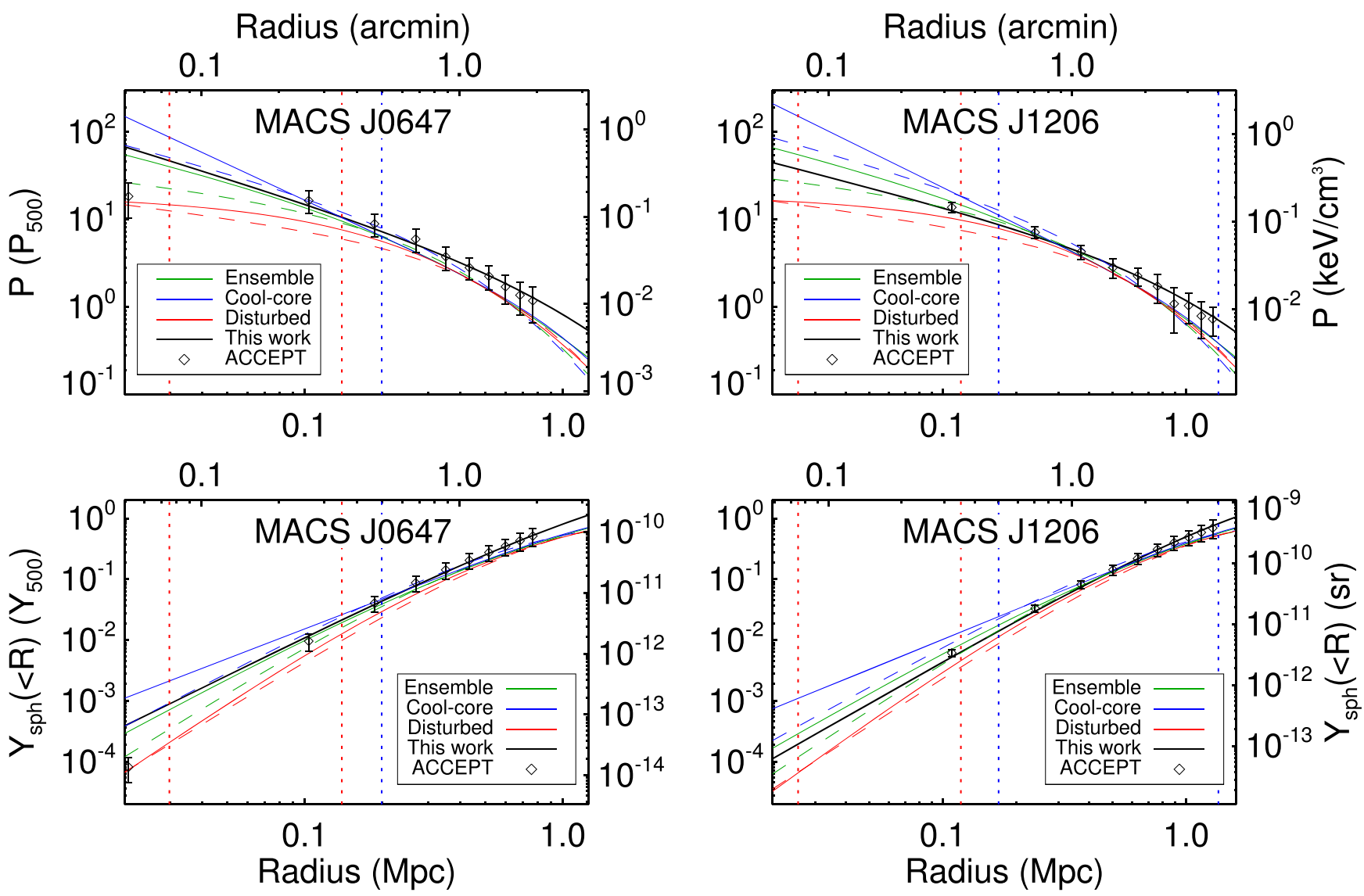

Figure 1. Pressure (upper) and spherically integrated Compton- $y$ (lower) profiles for MACS J0647.7+7015 (left) and MACS J1206.2-0847 (right). Dashed lines refer to the A10 sample of X-ray selected clusters, while solid lines correspond to the S13 sample including all of the CLASH clusters. For A10 and S13, respectively, "ensemble" (green) refers to the entire cluster sample, and profiles for cool-core (blue) and disturbed (red) morphologies are also separately shown. The X-ray derived pressure measurements from the ACCEPT database are plotted as diamonds. The best-fit Bolo+MUSTANG model presented in Section 5 is given by the solid black line in each plot. The vertical dotted lines surround the radial dynamic range (resolution to FOV) covered by MUSTANG (red) and Bolocam (blue). Note the Bolocam FOV extends beyond the radial range shown for MACS J0647.7+7015. The integrated Compton- $y$ profiles were computed according to Equations (3) and (6).

over statistically identical noise realizations. Given that our detector noise is dominated by phonon noise, pixel noise is largely uncorrelated, so we therefore take the noise covariance matrix $N$ to be diagonal. Residual atmospheric noise coupled with slight correlations between detectors will contribute offdiagonal elements to $N$. These terms are on average $3 \%$ of the magnitude of the diagonal terms and ignored in this procedure for computational simplicity. The best-fit amplitudes, corresponding to the minimum $\chi^{2}$, are then

$$
\boldsymbol{a}=\left(\boldsymbol{A}^{T} \boldsymbol{N}^{-1} \boldsymbol{A}\right)^{-1} \boldsymbol{A}^{T} \boldsymbol{N}^{-1} d
$$

The parameter uncertainties $\sigma^{2}\left(a_{k}\right)$ are given by the diagonal elements of the parameter covariance matrix $\left(A^{T} N^{-1} A\right)^{-1}$.

We perform the fits over a region within $1^{\prime}$ of the cluster centers. This scale is chosen to match the MUSTANG angular transfer function and we find that the results do not change significantly for fits using larger regions. Given the $1^{\prime \prime} \times 1^{\prime \prime}$ map pixels, this yields roughly $\pi(60)^{2}=11,310$ degrees of freedom (dof), minus the number of model components we include in each fit. The probability to exceed $\chi^{2}$ (PTE) reflects the probability that deviations between the given model and the data, at least as large as those observed, would be seen by chance, assuming the model is correct.

\subsection{Bolo+MUSTANG gNFW Profiles}

The Bolocam gNFW profiles are derived following the fitting procedure in Sayers et al. (2011) and Czakon et al. (2015), which we summarize briefly below.

First, the gNFW profile is used to obtain a three-dimensional model of the SZE. Next, this model is projected to twodimensions, scaled in angular size according to the cluster redshift, and convolved with both the Bolocam PSF and the transfer function of the Bolocam data processing. The result is then compared to the Bolocam image in order to obtain the best-fit parameters of the gNFW profile. For these fits, we use Equation (1) to convert the Bolocam brightness images to units of Compton- $y$. We include the relativistic corrections of Itoh et al. (1998) and Itoh \& Nozawa (2004), assuming the isothermal temperature given in Table 1.

Following the above procedure, we fit the Bolocam data with gNFW profiles spanning a range of fixed $\gamma$ values from 0 to 1.5. For generality, we fit elliptical models to the Bolocam data, although we note that these models produce axial ratios that are close to 1 (i.e., the elliptical models are nearly circular). For each profile, we assume the A10 "universal profile" values $\alpha=1.05$ and $\beta=5.49$. The normalization $P_{0}$, centroid, and scale radius $R_{\mathrm{s}}$ are allowed to float. These best-fit pressure profiles to Bolocam are shown in Figure 2. The integrated Compton- $y$ profiles are also shown. 

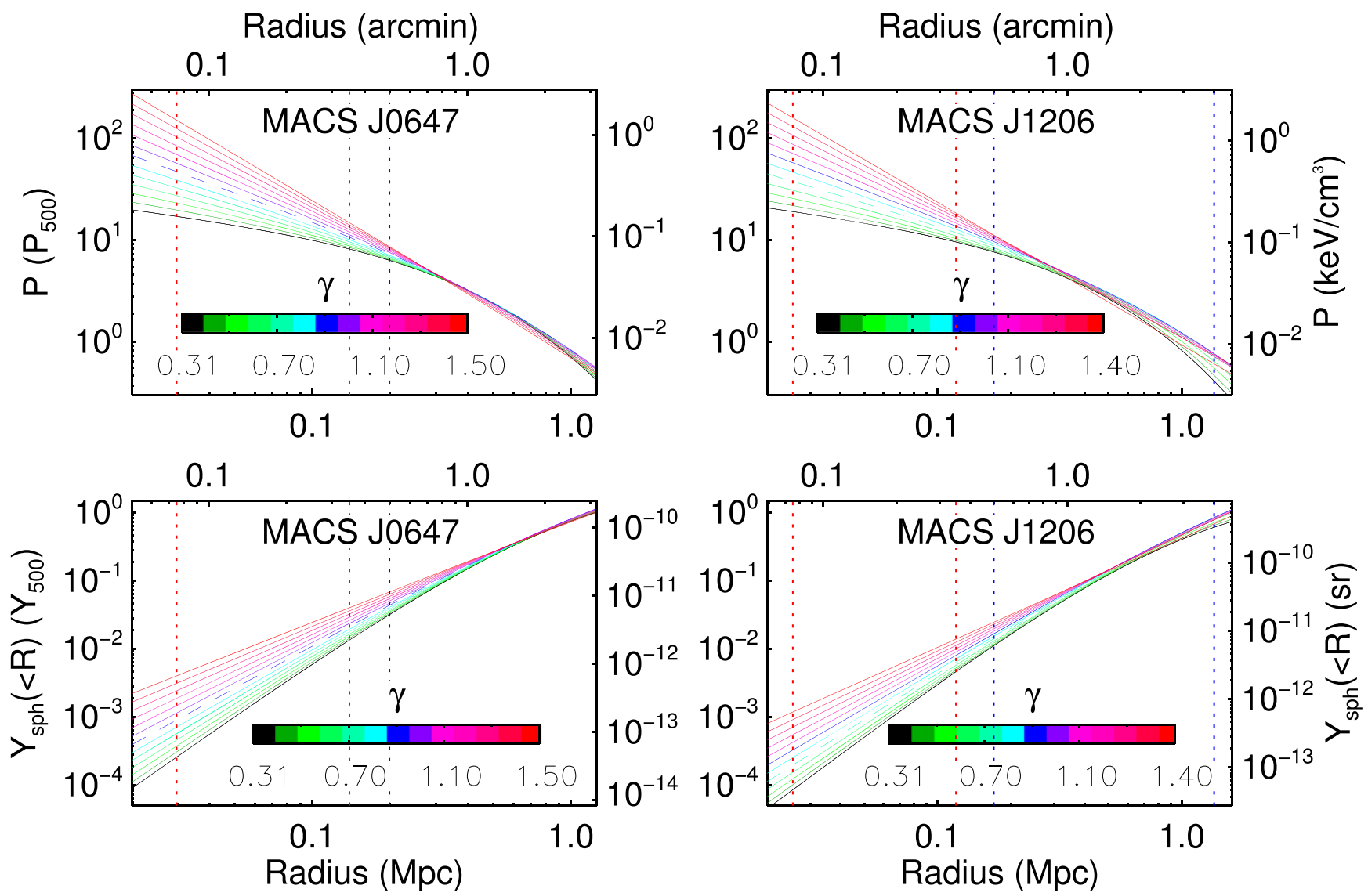

Figure 2. Pressure (upper) and spherically integrated Compton- $y$ (lower) profiles generated from fits of generalized NFW profiles to Bolocam measurements of MACS J0647.7+7015 and MACS J1206.2-0847, in the left and right columns, respectively. Each profile represents the gNFW that best fits the Bolocam data given a fixed value of $\gamma$, represented by the color bars, with $\alpha$ and $\beta$ held at the A10 values. In general, Bolocam has the largest constraining power between $1^{\prime}$ and $3^{\prime}$ in radius, and all of the models overlap to a high degree in this radial region. This highlights the inherent parameter degeneracies between $P_{0}, R_{\mathrm{s}}$, and $\gamma$ in the gNFW model, which can be broken using the high-resolution MUSTANG data. The dashed lines correspond to the best fit Bolo+MUSTANG models, which have $\gamma=0.9$ for MACS J0647.7+7015, and $\gamma=0.7$ for MACS J1206.2-0847 (see Table 3). From left to right, the vertical dotted lines mark the resolution and FOV, respectively, of MUSTANG (red) and Bolocam (blue), as in Figure 1.

We compare each of these models to the MUSTANG data as described in Section 4.1. We choose a grid over $\gamma$ values because $\gamma$ defines the inner slope of the ICM profile where we expect MUSTANG to be most sensitive. From the grid of bestfit models to the Bolocam data, the model with the best fit to the MUSTANG data is selected as the overall best fit, referred to as the Bolo+MUSTANG model. We emphasize that this is a serial-fitting procedure, in which we first fit a set of gNFW models to the Bolocam data, independently of the MUSTANG observations, and then select a single model from this set that best fits the MUSTANG data. Effectively, the Bolocam data first constrain the values of $P_{0}$ and $R_{\mathrm{S}}$ (for fixed $\gamma, \alpha$, and $\beta$ ), and then the MUSTANG data constrain the value of $\gamma$.

\section{RESULTS}

\subsection{MACS J0647.7+7015}

MACS J0647.7+7015, discovered during the Massive Cluster Survey (MACS; Ebeling et al. 2001, 2007), is a seemingly relaxed massive system at $z=0.591$, but contains multiple cD galaxies (see Hung \& Ebeling 2012), which may indicate ongoing merger activity (Mann \& Ebeling 2012). Figure 3 shows a composite image of MACS J0647.7 +7015 including optical, strong-lensing, and X-ray images.
The mass distribution from the strong-lensing analysis (Zitrin et al. 2011) is doubly peaked and elongated in the $E-W$ direction. The X-ray emission measured by Chandra shows similar elongation as does the SZE flux measured by MUSTANG.

The MUSTANG map of MACS J0647.7+7015 is shown in Figure 4 . The peak SZE flux is $-121 \pm 16 \mu \mathrm{Jy}_{\text {beam }}{ }^{-1}$. The measured decrement $(>3 \sigma)$ encompasses an elongated region approximately $25^{\prime \prime} \times 38^{\prime \prime}$. The total SZE flux measured by MUSTANG, within the region with $>3 \sigma$ significance of the decrement, is $-535 \pm 38 \mu \mathrm{Jy}$.

Figure 5 shows the pseudo- $y$ template derived from X-ray measurements according to Equation (4). We normalize the integrated pseudo Compton- $y$ based on the Bolocam flux as described in Section 3.

Following the procedure outlined in Section 3, we determine the thermal SZE model that best simultaneously describes the MUSTANG and Bolocam data to be a gNFW profile with

$$
\left[P_{0}, R_{\mathrm{s}}(\mathrm{Mpc}), \gamma, \alpha, \beta\right]=[0.54,4.34,0.90,1.05,5.49],
$$

with a ratio between major and minor axes of 1.27 and position angle $-174^{\circ} \mathrm{E}$ of $\mathrm{N}$, hereafter referred to as the $\gamma=0.9$, or 


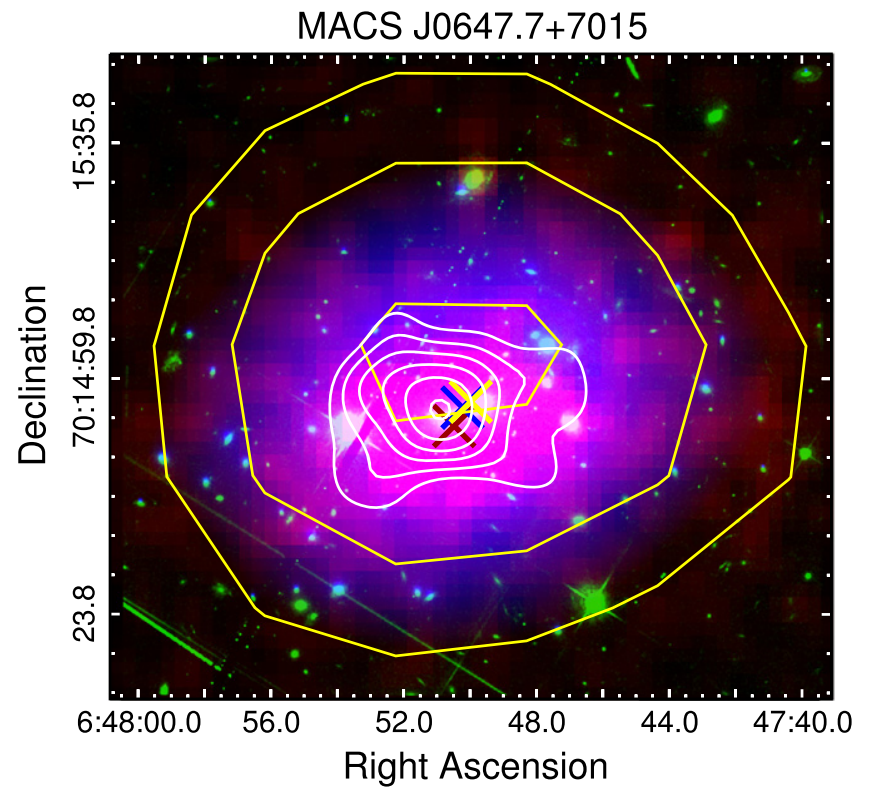

Figure 3. Composite image of MACS J0647.7+7015. Green is HST, blue is the total mass distribution derived from strong gravitational lensing (Zitrin et al. 2011), and red is X-ray surface brightness measured by Chandra. MUSTANG S/N contours from Figure 4 are overlaid in white and Bolocam contours $(\mathrm{S} / \mathrm{N} \approx[12,13,14])$ are overlaid in yellow. Although the Bolocam peak is located slightly north of the cluster center, there is good agreement in general between the X-ray, SZE, and lensing mass distributions. Crosses denote the centroid for the X-ray surface brightness (dark red), BCGs (blue), and Bolocam SZE (yellow). MACS J0647.7+7015 exhibits an elliptical morphology with two distinct $\mathrm{cD}$ galaxies, which may indicate merger activity, but otherwise appears to be relaxed. The blue cross above is centered between the two cD galaxies.

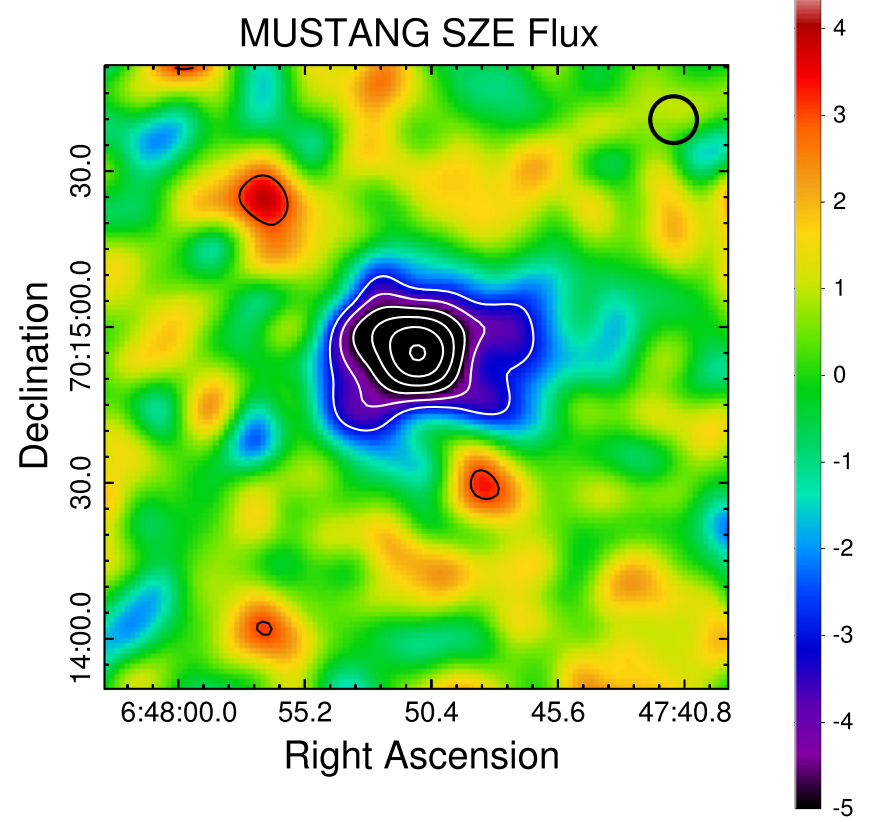

Figure 4. MUSTANG SZE S/N map of MACS J0647.7+7015 smoothed with the $9^{\prime \prime}$ beam represented by the black circle in the upper right. Contours are shown in increments of $1-\sigma$ beginning at $3 \sigma$ for SZE decrement (white) and positive flux (black).

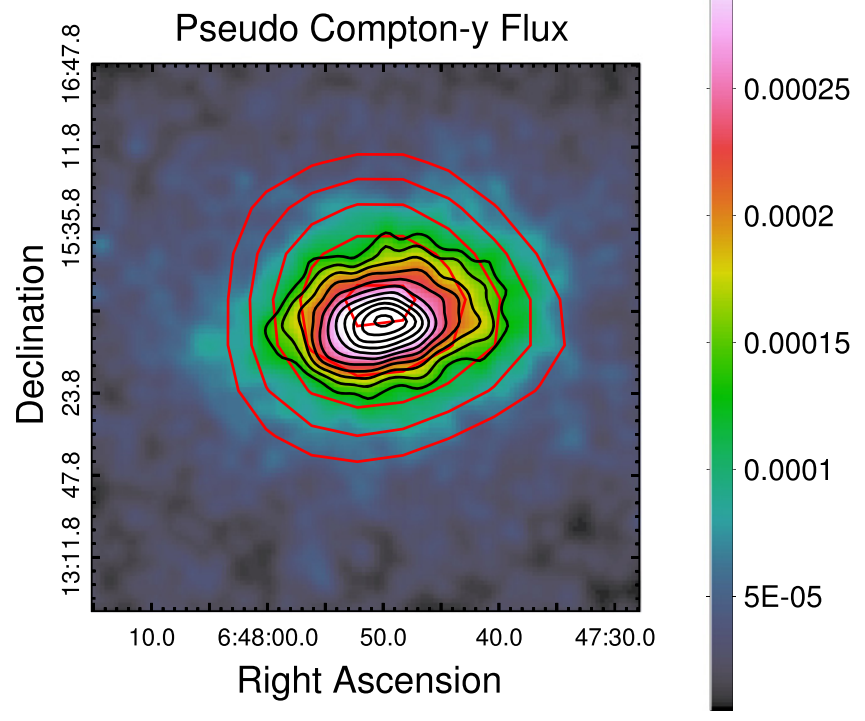

Figure 5. MACS J0647.7+7015 X-ray derived Compton-y map assuming an isothermal temperature of $11.5 \mathrm{keV}$ and effective depth $\ell=1.4 \mathrm{Mpc}$. The contours are shown for X-ray pseudo-y (black) and Bolocam data (red) in increments of $0.14 \times 10^{-4}$ beginning at $1.3 \times 10^{-4}$ for both. The Bolocam PSF smooths the signal significantly on the scale of this image, which explains the broader contours relative to the X-ray.

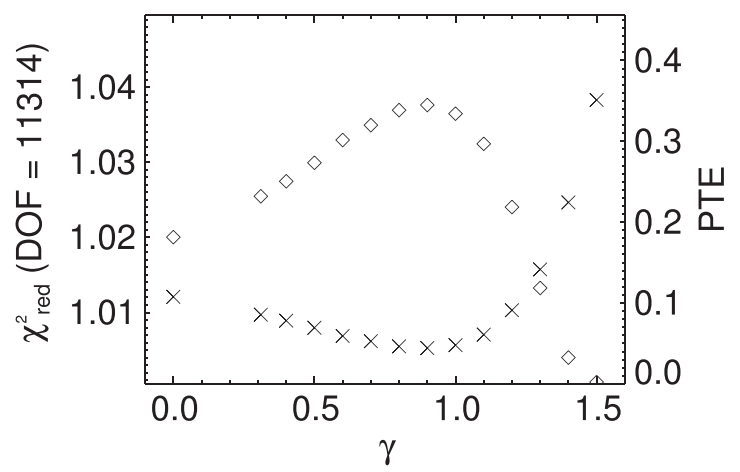

Figure 6. Goodness of fit parameters $\chi_{\text {red }}^{2}$ (crosses) and PTE (diamonds) from the comparison between MUSTANG data and the Bolocam-derived models for MACS J0647.7+7015. We determine the best-fit model to be a gNFW with $\gamma=0.90$, yielding $\chi^{2} /$ dof $=11374 / 11314$ and PTE $=0.34$.

$\mathrm{gNFW}_{\gamma=0.9}$, model. $R_{\mathrm{S}}$ is computed from the geometric mean of the major and minor axes. Figure 6 shows the calculated reduced $\chi^{2}\left(\chi_{\text {red }}^{2}=\chi^{2} /\right.$ dof $)$ and PTE as a function of the fixed $\gamma$ value. The $\mathrm{gNFW}_{\gamma=0.9}$ model gives $\chi^{2} /$ dof $=11378 / 11314$ with PTE $=0.34$ (see Table 4).

The X-ray pseudo-SZE and $\mathrm{gNFW}_{\gamma=0.9}$ model for MACS J0647.7+7015, after being filtered through the MUSTANG pipeline, are shown in Figure 7. Also shown are the azimuthally averaged radial profiles. The X-ray flux is concentrated on smaller scales and passes through the MUSTANG pipeline with less attenuation compared to the gNFW models, which have shallower profiles extending to larger radii. The filtered $\mathrm{gNFW}_{\gamma=0.9}$ flux peak is offset slightly north of the X-ray peak. The radially averaged profiles from the filtered maps are fairly consistent between all three data sets. 
Table 4

Summary of Fit Results

\begin{tabular}{lccr}
\hline \hline Cluster & Model & $\chi^{2} /$ dof & PTE \\
\hline MACS J0647.7+7015 & & & \\
\hline & A10 & $11425 / 11314$ & 0.23 \\
& gNFW $_{\gamma=0.9}$ & $11378 / 11314$ & 0.34 \\
& Pseudo-SZE & $11497 / 11314$ & 0.11 \\
\hline MACS J1206.2-0847 & & & \\
& & & 0.68 \\
& A10 & $11237 / 11307$ & 0.70 \\
& gNFW & & \\
& & $11227 / 11307$ & 0.25 \\
& Pseudo-SZE & $11408 / 11307$ & \\
\hline
\end{tabular}

Note. Fit results for the A10, gNFW, and pseudo-SZE models. The fits for MACS J1206.2-0847 included a model for the point source with a floating amplitude.

\subsubsection{Discussion}

In MACS J0647.7+7015, we find good agreement between the MUSTANG high-resolution SZE image and the X-ray and Bolocam measurements. We summarize the results from the fitting procedure in Table 4 . The $\gamma=0.9 \mathrm{gNFW}$ model best fits the MUSTANG data with a PTE of 0.34, whereas the A10 and pseudo-SZE are less favored (PTE $\leqslant 0.23$ ).

The compact positive sources in Figure 4 are significant $(>3 \sigma)$ even after accounting for the lower observing coverage outside the cluster core, however, we find no counterparts for these sources in any other data set. In computing the significances we have assumed that the MUSTANG mapdomain noise follows a Gaussian distribution within a 2 ' radius, which we verified by inspecting the histogram of the noise map for MACS J0647.7+7015. High resolution radio observations were not obtained for MACS J0647.7+7015 so spectral coverage close to $90 \mathrm{GHz}$ is limited. We take jackknives of the data, split into four equal integration times, and the sources appear with similar flux in each segment, which is unlikely for an artifact. Therefore, it is possible that these are yet unidentified objects such as lensed high- $z$ dusty galaxies or shallow spectrum AGNs, which may be confirmed by future observations with high resolution coverage near $90 \mathrm{GHz}$.

\subsection{MACS J1206.2-0847}

MACS J1206.2-0847 is a mostly relaxed system at $z=0.439$ that has been studied extensively through X-ray, SZE, and optical observations (e.g., Ebeling et al. 2001, 2009; Gilmour et al. 2009; Umetsu et al. 2012; Zitrin et al. 2012; Biviano et al. 2013; S13). A composite image with the multiwavelength data is shown in Figure 8. We include high resolution $610 \mathrm{MHz}$ data from the Giant Metrewave Radio Telescope (GMRT; project code 21_017). These data reveal extended radio emission to the west of the $\sim 0.5 \mathrm{Jy}$ central AGN.

The MUSTANG SZE map of MACS J1206.2-0847 is shown in Figure 9. The majority of the SZE decrement extends to the northeast and is contaminated by emission from the central AGN.

The X-ray pseudo- $y$ and SZE decrement measured by Bolocam are shown in Figure 10.
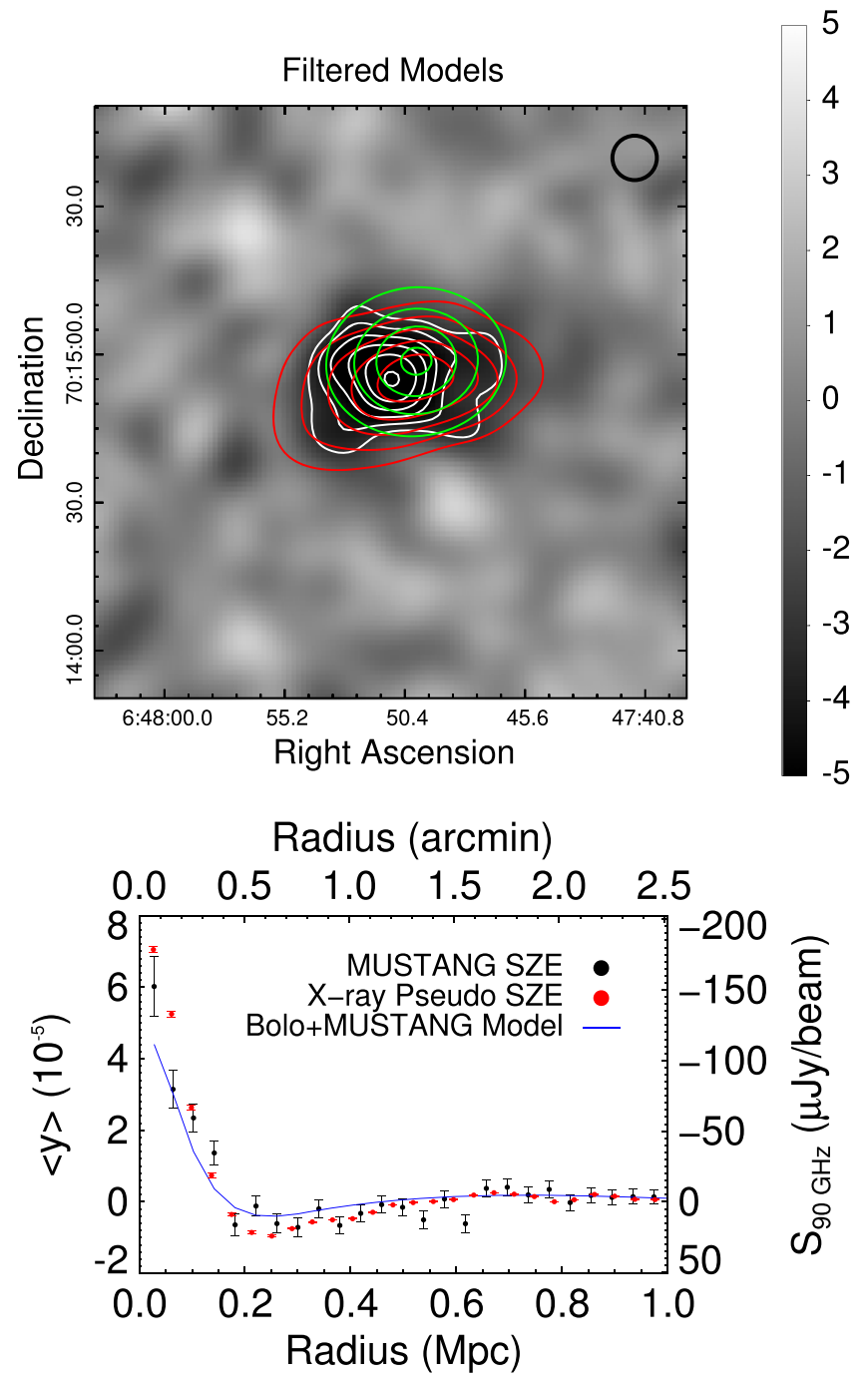

Figure 7. MACS J0647.7+7015 pseudo SZE map derived from Chandra $\mathrm{X}$-ray data (top, red contours) with green contours representing the Bolo + MUSTANG $\mathrm{gNFW}_{\gamma=0.9}$ model from this work. The MUSTANG transfer function has been applied to both and the MUSTANG PSF is shown as a black circle. The white contours are MUSTANG S/N from Figure 4. The red and green contours are overlaid in units of $-50 \mu \mathrm{Jy}_{\text {beam }}{ }^{-1}$ starting at $-50 \mu \mathrm{Jy} \mathrm{beam}^{-1}$. Azimuthally averaged radial profiles are shown in the lower panel. Aside from the central $\sim 0.1 \mathrm{Mpc}$ where the X-ray and SZE flux are sharply peaked, the radially averaged flux from MUSTANG closely follows both the $\mathrm{gNFW}_{\gamma=0.9}$ model and the X-ray pseudo SZE flux.

The BCG $\left(\alpha_{\mathrm{J} 2000}=12^{\mathrm{h}} 06^{\mathrm{m}} 12^{\mathrm{s}} 1, \delta_{\mathrm{J} 2000}=-08^{\circ} 48^{\prime} 3^{\prime \prime}\right)$ in MACS J1206.2-0847 harbors a radio-loud AGN that is detected by MUSTANG at high significance $(\mathrm{S} / \mathrm{N}>4)$. Using a spatial template derived from the MUSTANG map, we construct a compact source model and allow the amplitude to float in the joint fits with bulk SZE models, in order to account for the degeneracy between the co-spatial positive emission and SZE decrement. AGN brightness is generally represented as a power law with frequency, given by

$$
\log (S(\nu)[\mathrm{mJy}]=\alpha \log (\nu[\mathrm{MHz}]))+\beta
$$

where $\alpha$ is the spectral index and $\beta$ is the abscissa. Extrapolating from low frequency $(\nu<1.4 \mathrm{GHz})$ measurements, SPECFIND V2.0 (Vollmer et al. 2010) predicts $\alpha=-1.26 \pm 0.1$ and $\beta=6.2 \pm 0.2$, or a $90 \mathrm{GHz}$ flux of 


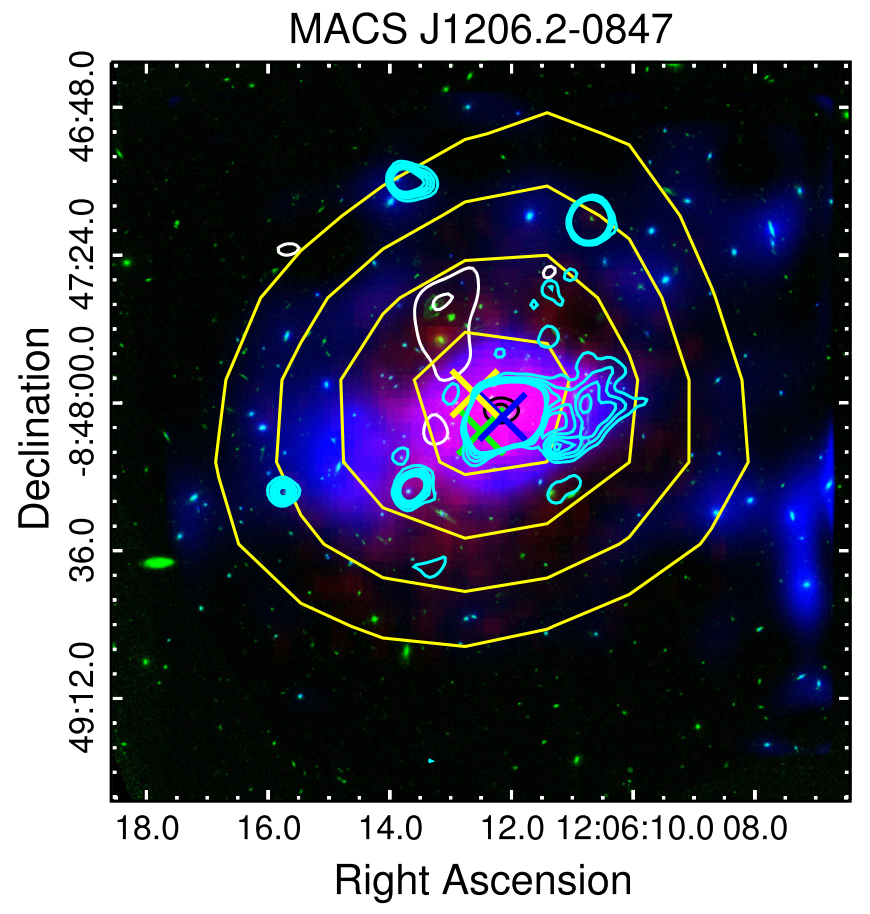

Figure 8. Composite image of MACS J1206.2-0847. Green is HST, blue is the total mass distribution derived from strong gravitational lensing (Zitrin et al. 2012), and red is X-ray surface brightness measured by Chandra. MUSTANG S/N contours from Figure 9 are overlaid in white (negative) and black (positive). Bolocam contours $(\mathrm{S} / \mathrm{N} \approx[15,17,19,21])$ are overlaid in yellow. Radio contours from GMRT $610 \mathrm{MHz}$ observations are overlaid in cyan, and span $8 \sigma$ to $17 \sigma$ in steps of $3 \sigma$. The crosses denote the centroids from the Bolocam data (yellow), the diffuse X-ray distribution (green), and the BCG (blue). The offsets between these centroids, as well as the extended radio emission, could be indicative of a disturbed cluster morphology.

$S_{90}=879 \pm 253 \mu \mathrm{Jy}$. By way of comparison, our joint fit results give $S_{90}=584-765 \mu \mathrm{Jy}$, summarized in Table 5 .

Figure 11 shows the goodness of fit statistics for the gNFW + point source model fitting. With $\chi_{\text {red }}^{2}=0.993$ and $\mathrm{PTE}=0.70$, the best fit model is a gNFW with

$$
\left[P_{0}, R_{\mathrm{s}}(\mathrm{Mpc}), \gamma, \alpha, \beta\right]=[1.13,3.93,0.70,1.05,5.49],
$$

with a ratio between major and minor axes of 1.02 and position angle $-13^{\circ} \mathrm{E}$ of $\mathrm{N}$, hereafter $\mathrm{gNFW}_{\gamma=0.7}$ (see Table 4). The filtered $\mathrm{gNFW}_{\gamma=0.7}$ and pseudo-SZE models are shown in Figure 12. The Bolocam model is much more extended than the $\mathrm{X}$-ray and is subsequently filtered the most by the MUSTANG transfer function. The pseudo-SZE model shows a much higher peak after filtering, but diminishes rapidly with radius.

After subtracting the point source and gNFW ${ }_{\gamma=0.7}$ model, we find a $3 \sigma$ residual feature in MACS J1206.2-0847 (see Figure 9). The $3 \sigma$ contour encompasses a $73 \operatorname{arcsec}^{2}\left(2 \mathrm{kpc}^{2}\right)$ region with an integrated flux of $-61 \pm 21 \mu \mathrm{Jy}$. Using Equation (1) we calculate the integrated Compton- $y$, $Y_{\mathrm{SZ}} D_{A}^{2}=7.3 \times 10^{-7} \mathrm{Mpc}^{2}$ (see Table 6).

\subsubsection{Discussion}

The Bolo+MUSTANG $\mathrm{gNFW}_{\gamma=0.7}$ model for MACS J1206.2-0847 provides a good fit to the MUSTANG data when a point source model for the central AGN is included. However, since the point source is co-spatial with the SZE decrement and we allow the amplitude to float, a model with a steeper core slope will compensate with a stronger point source. Relative to MACS J0647.7+7015, in which MUSTANG does not detect AGN emission, this effect reduces the constraining power on $\gamma$, which can be seen by comparing Figures 6 and 11. Measurements of the point source flux closer in frequency to $90 \mathrm{GHz}$ are required to model and remove the source prior to fitting and thereby improve the constraining power on $\gamma$.

Previous analyses of MACS J1206.2-0847 suggest that the system is close to being in dynamical equilibrium. Gilmour et al. (2009) classify the cluster as visually relaxed based on its $\mathrm{X}$-ray morphology. The mass profiles derived from galaxy kinematics (Biviano et al. 2013), X-ray surface brightness, and combined strong and weak-lensing (Umetsu et al. 2012) are all consistent, which indicates that the system is likely relaxed.

As described in Section 5.2, MUSTANG detects an excess residual of SZE flux $(>3 \sigma)$ to the NE of the bulk ICM in MACS J1206.2-0847, after removing the point source and $\mathrm{gNFW}_{\gamma=0.7} \mathrm{SZE}$ models. This signal does not appear to have a counterpart in the X-ray surface brightness image, nor is there a diffuse radio feature in GMRT observations that would point to a shock associated with an energetic merger event (e.g., Ferrari et al. 2011). When comparing the MUSTANG map to the optical image and a weak lensing mass reconstruction using data and methods presented in Umetsu et al. (2012), we do however see some evidence that this source is aligned with a filamentary structure to the N-NE (Figure 13).

Figure 13 shows an optical image of MACS J1206.2-0847 with weak lensing mass contours overlaid. The SE elongation in the mass distribution follows a filamentary structure that has been noted in previous analyses (see Umetsu et al. 2012; Annunziatella et al. 2014). Additionally, there appears to be an elongation in the mass distribution to the NE, in the direction of the feature detected by MUSTANG. The centroid of the SZE signal measured by Bolocam is also shifted to the NE (see Figure 8).

\subsubsection{Galaxy Group Scenario}

We consider the case of a galaxy group leading to the SZE feature detected by MUSTANG. Using the residual flux measured in the filtered, model-subtracted MUSTANG SZE maps, we can place constraints on the group mass. By simulating a suite of idealized A10 cluster Compton- $y$ maps, we find the MUSTANG residual can only provide a lower limit to the mass, since the filtering effects remove an unknown and possibly large SZE flux component from angular scales inaccessible to MUSTANG, while for a small enough group or cluster little flux is filtered. We use this mass to infer what the X-ray surface brightness of the group would be, and determine if such a lower limit is consistent the upper limit placed by X-ray. The residual integrated SZE flux of $-61 \mu \mathrm{Jy}$ corresponds to a mass lower limit of $M_{500}>1.3 \times 10^{13} M_{\odot}$ and soft (0.1-2.4 keV) X-ray luminosity of $L_{X}>7.99 \times 10^{43}$ $\mathrm{erg} \mathrm{s}^{-1}$ (see Table 6). In this calculation we have assumed the $Y-M$ and $Y-L_{\mathrm{X}}$ scaling relations given in A10. We note that $Y_{\mathrm{SZ}}$ $D_{A}^{2}=9.53 \times 10^{-8} \mathrm{Mpc}^{2}$ is below the mass limit of the sample used in A10, so this is an extrapolation.

Using the spectroscopic redshifts of Biviano et al. (2013), which are part of the "CLASH-Very Large Telescope (VLT)" VIsible MultiObject Spectrograph (VIMOS) Large Programme and have been recently made publicly available, we analyze 


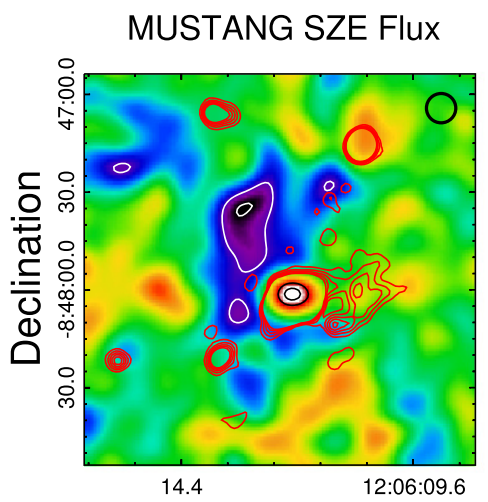

Right Ascension

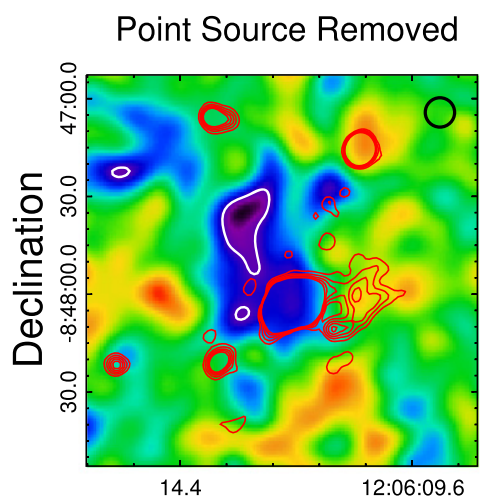

Right Ascension

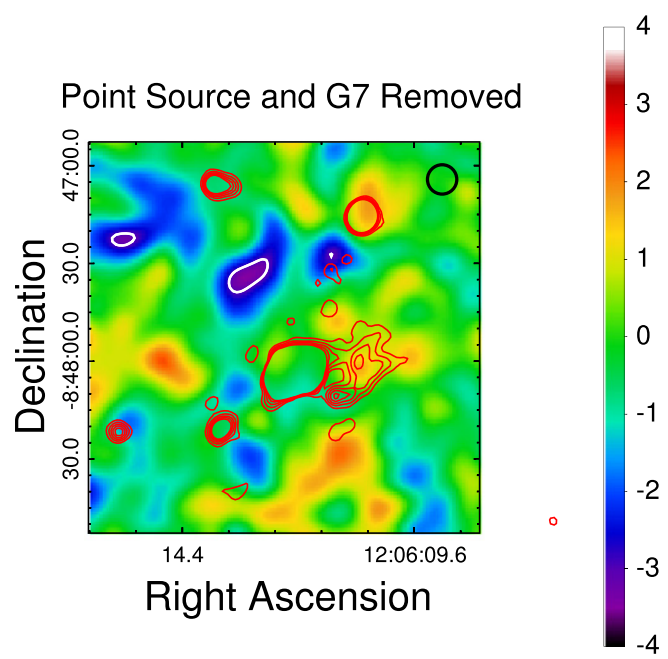

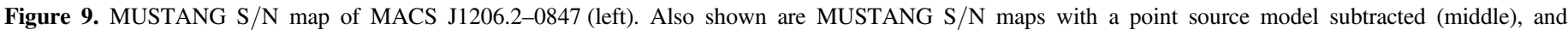

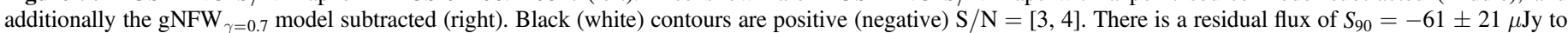

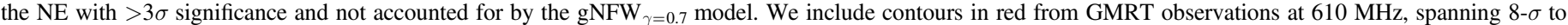
$17 \sigma$ in steps of $3 \sigma$. In each panel, the $9^{\prime \prime}$ MUSTANG beam is drawn as a black circle in the upper right.

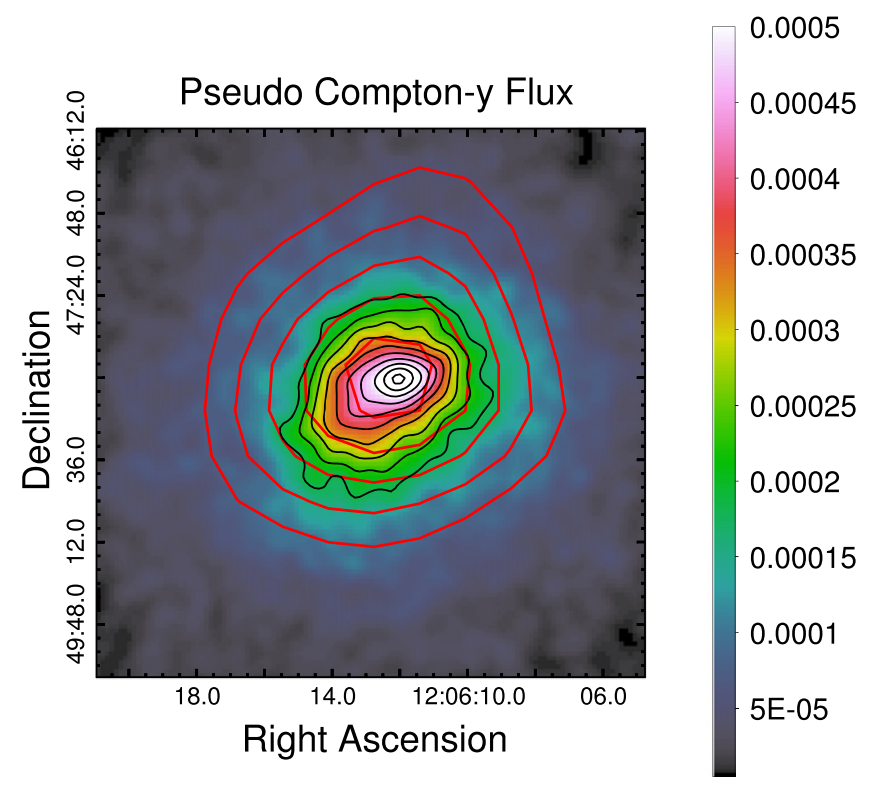

Figure 10. MACS J1206.2-0847 X-ray derived Compton- $y$ map assuming an isothermal temperature of $10.7 \mathrm{keV}$ and effective depth $\ell=2.0 \mathrm{Mpc}$. The contours are shown for X-ray pseudo-y (black) and Bolocam data (red) in increments of $0.25 \times 10^{-4}$ beginning at $1.7 \times 10^{-4}$ for both. As in Figure 5, the Bolocam contours are broader than the X-ray, due to the smoothing of the core flux by the Bolocam PSF.

galaxy structures outside the main cluster peak in the redshift distribution, selecting galaxies corresponding to foreground and background peaks. One of these redshift bins, at $z \sim 0.42$, contains 13 galaxies that are located near the SZE peak. We take these galaxies to be members of a potential group associated with the SZE feature and compute the line of sight velocity dispersion, $\sigma_{\mathrm{V}}$. For this group we find $\langle z\rangle=0.423$ and $\sigma_{\mathrm{V}}=650 \mathrm{~km} \mathrm{~s}^{-1}$. Therefore, this is potentially either a foreground group $\approx 100 \mathrm{Mpc}$ in front of the cluster or a group falling into the cluster with a rest frame velocity of $V_{\mathrm{rf}} \approx 3500 \mathrm{~km} \mathrm{~s}^{-1}$ toward the observer.
Table 5

Point Source Flux and Extrapolated Spectral Indices

\begin{tabular}{lccc}
\hline \hline Model & $\begin{array}{c}S_{90} \\
(\mu \mathrm{Jy})\end{array}$ & $\alpha$ & $\beta$ \\
\hline SPECFIND & $879 \pm 253$ & $-1.26 \pm 0.09$ & $6.19 \pm 0.24$ \\
A10 & $674 \pm 61$ & $-1.32 \pm 0.05$ & $6.34 \pm 0.25$ \\
gNFW $_{\gamma=0.7}$ & $765 \pm 61$ & $-1.28 \pm 0.05$ & $6.25 \pm 0.24$ \\
Null & $584 \pm 61$ & $-1.35 \pm 0.05$ & $6.45 \pm 0.25$ \\
\hline
\end{tabular}

Notes. Point source fluxes derived from joint fits with bulk SZE models. The first row provides the flux at $90 \mathrm{GHz}\left(S_{90}\right)$ extrapolated from measurements at lower frequencies $(74-1400 \mathrm{MHz})$ given in the SPECFIND V2.0 catalog (Vollmer et al. 2010). The A10 model refers to the ensemble parameters given in Table 3. The $\mathrm{gNFW}_{\gamma=0.7}$ model is the best-fit Bolo+MUSTANG model from this work. The "null" model assumes there is no SZE decrement coincident with the point source. This represents a lower limit on the flux at $90 \mathrm{GHz}$ and and therefore the steepest (most negative) likely spectral index.

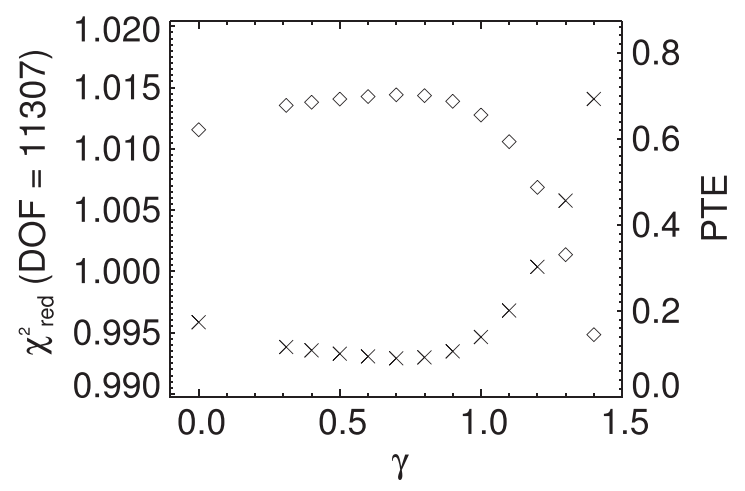

Figure 11. Goodness of fit parameters $\chi_{\text {red }}^{2}$ (crosses) and PTE (diamonds) from the comparison between MUSTANG data and the Bolocam-derived models for MACS J1206.2-0847. We determine the best-fit model to be a gNFW $(\gamma=0.70)$, for which we calculate $\chi^{2} / \operatorname{dof}=11227 / 11307$ and $\mathrm{PTE}=0.70$.

Following the $\sigma_{\mathrm{V}}-M_{200}$ relation of Munari et al. (2013), we compute a group mass of $M_{200}=(2.4 \pm 1.5) \times 10^{14} M_{\odot}$ within $R_{200} \sim 1.1 \mathrm{Mpc}$, corresponding to $M_{500}=1.4 \pm$ $0.9 \times 10^{14} M_{\odot}$ and $R_{500}=0.7_{-0.2}^{+0.1} \mathrm{Mpc}$ for a typical scaling of $M_{500}=0.6 \times M_{200}$ of an NFW mass profile. 


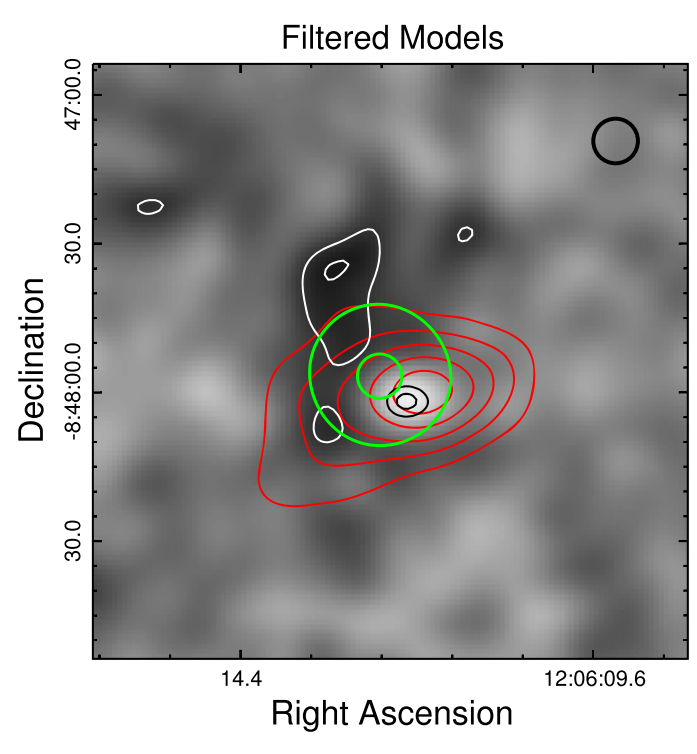

Radius (arcmin)

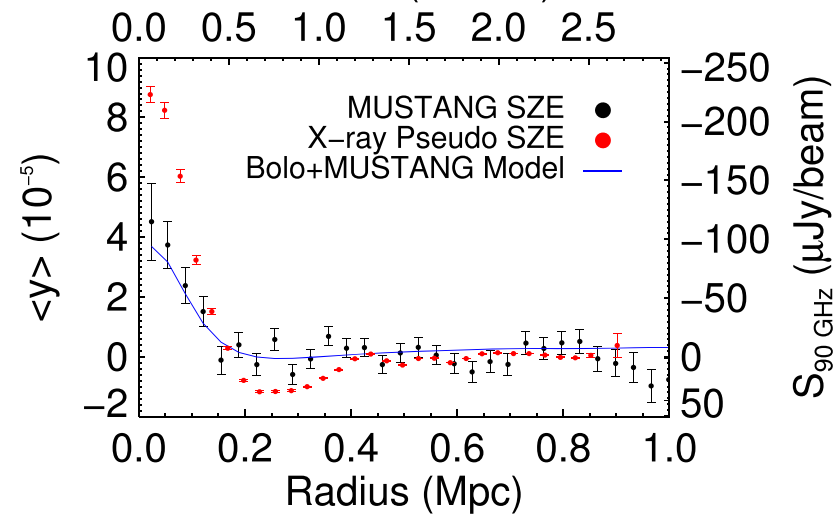

Figure 12. X-ray-derived (top, red contours) and Bolo+MUSTANG gNFW $\gamma=0.7$ model (top, green contours) for MACS J1206.2-0847, each with the MUSTANG transfer function applied. Both have been smoothed with the MUSTANG PSF given by the black circle. The red and green contours start at $-75 \mu \mathrm{Jy}_{\text {beam }}{ }^{-1}$ and increase in steps of $-25 \mu \mathrm{Jy}_{\text {beam }}{ }^{-1}$. The MUSTANG $\mathrm{S} / \mathrm{N}$ contours from Figure 9 are overlaid in white. Azimuthally averaged radial profiles are shown in the lower panel. The filtered X-ray derived flux shows a sharper peak relative to the Bolocam and MUSTANG data, which could be a result of the way in which the pseudo-y map is normalized (see Figure 10).

Table 6

MACS J1206.2-0847 SZE Residual Flux and Lower Mass Limits after Cluster Model and Point Source Subtraction

\begin{tabular}{lcccc}
\hline \hline $\begin{array}{l}\text { Model(s) } \\
\text { Removed }\end{array}$ & $\begin{array}{c}S_{90} \\
(\mu \mathrm{Jy})\end{array}$ & $\begin{array}{c}Y_{\mathrm{SZ}_{\mathrm{Z}} D_{A}{ }^{2}} \\
\left(10^{-8} \mathrm{Mpc}^{2}\right)\end{array}$ & $\begin{array}{c}M_{500}{ }^{\mathrm{a}} \\
\left(10^{13} M_{\odot}\right)\end{array}$ & $\begin{array}{c}L_{\mathrm{X}}{ }^{\mathrm{b}} \\
\left(10^{43} \mathrm{erg} \mathrm{s}^{-1}\right)\end{array}$ \\
\hline Point Src $^{\mathrm{c}}$ & $-193 \pm 36$ & $32 \pm 6$ & $2.6 \pm 1.0$ & $3.0 \pm 1.7$ \\
gNFW $_{\gamma=0.7}$ & $-61 \pm 21$ & $9.5 \pm 3.3$ & $1.3 \pm 0.7$ & $1.7 \pm 1.3$ \\
$\quad+\mathrm{Pt} \mathrm{Src}$ & & & & \\
\hline
\end{tabular}

Notes. Integrated flux estimates from the MUSTANG map. The first row corresponds to the total SZE flux with the point source emission taken into account. The bottom row is the residual flux after removing the best-fit gNFW $\gamma=0.7$ ICM model in addition to the point source flux. The integrated fluxes were computed within the regions enclosed by the $3 \sigma$ contours shown in the right panel of Figure 9.

${ }^{\mathrm{a}, \mathrm{b}} M_{500}$ and $L_{\mathrm{X}}$ are derived from the A10 $Y_{\mathrm{SZ}}-M_{500}$ and $M_{500}-L_{\mathrm{X}}$ scaling relations.

${ }^{\mathrm{c}}$ We use the $-765 \mu \mathrm{Jy}$ point source model from Table 5 .

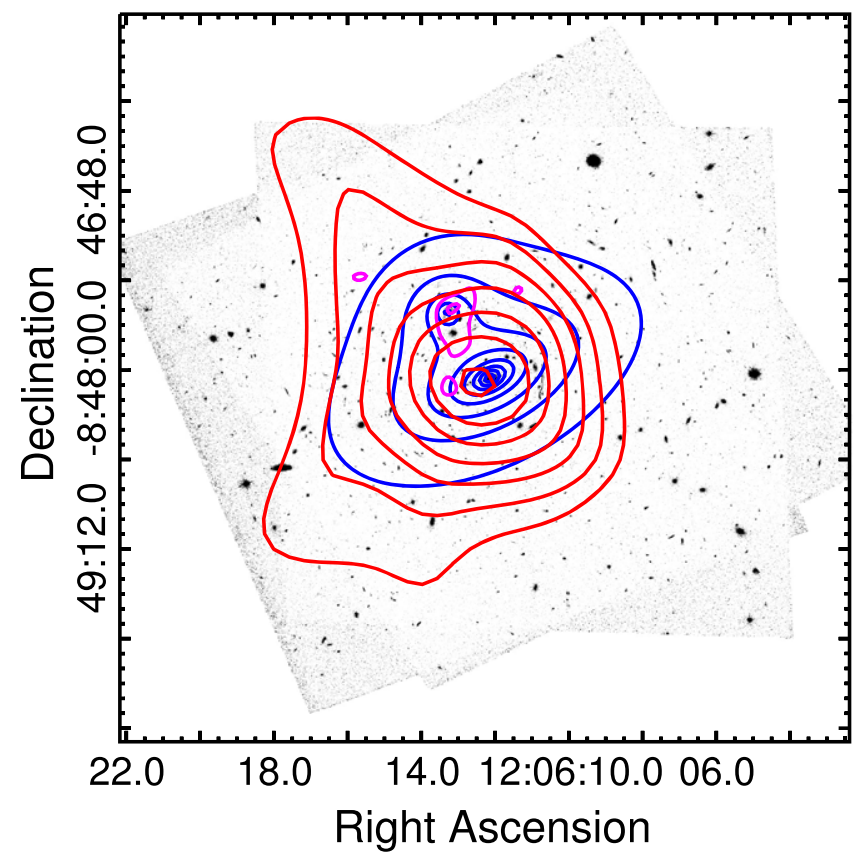

Figure 13. Optical image from HST (grayscale) overlaid with the weak-lensing mass distribution (red) from Umetsu et al. (2012), the MUSTANG S/N contours (magenta), and the best fit eNFW+NFW two-halo model (blue). In addition to the $\mathrm{E}-\mathrm{W}$ elongation noted in previous observations, there is an elongation to the NE. This suggests that the MUSTANG SZE detection may correlate with real structure such as an infalling galaxy group.

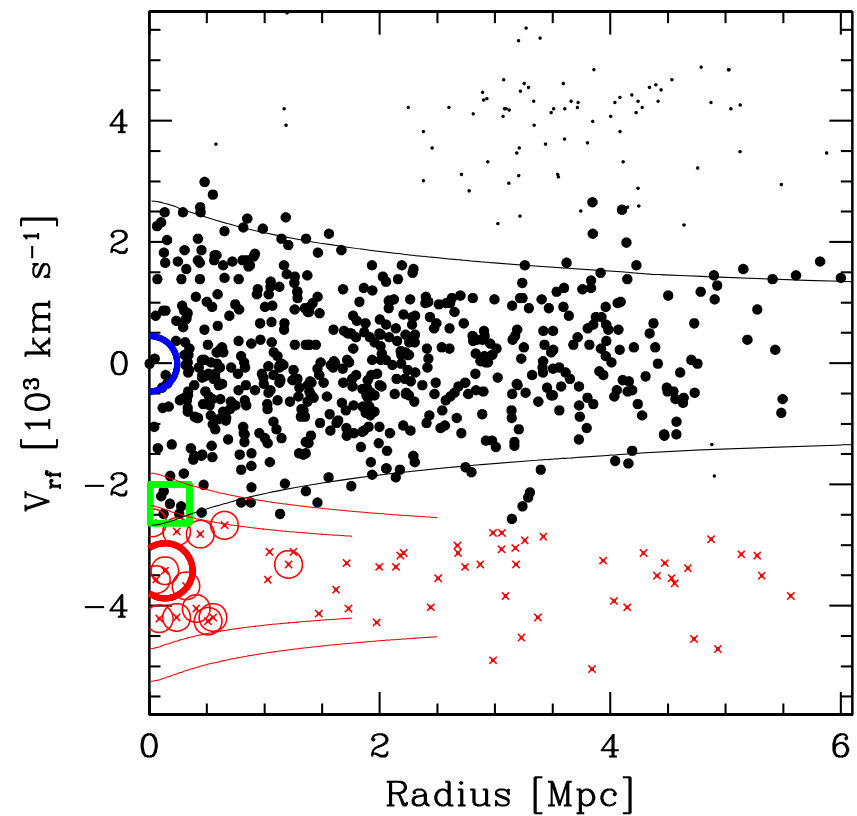

Figure 14. Rest-frame line of sight velocity vs. projected distance from the center of MACS J1206.2-0847, based on the CLASH-VLT VIMOS survey. The cluster center coincides with the position of its BCG (blue circle at 0,0 ). Solid black circles indicate cluster members (see also Figure 2-lower panel of Biviano et al. 2013). Small red crosses indicate galaxies corresponding to the $z \sim 0.42$ peak in the redshift distribution and red circles highlight the 13 galaxies within $1.1 \mathrm{Mpc}$ of the SZE peak, that are the likely members of the putative group within $R_{200}$. The black and red curves show the limits due to the escape velocity in the cluster and the group, respectively. For the group we show both sets of curves corresponding to $M_{200} \pm 1 \sigma$ error limits out to $2 R_{200}$. The blue circle coincides with the cluster BCG, the red circle marks the brightest galaxy in the group which is located at the SZE peak, and the green square is a spiral galaxy that lies on the boundary between the cluster and the potential group. 
Figure 14 shows the projected phase space diagram for the galaxies in this study including escape velocity curves for both the primary cluster and the potential group. We compute the escape velocities using an NFW mass density profile and the procedure of den Hartog \& Katgert (1996). The escape velocity curves for the cluster and the group are centered on the BCG and the brightest group galaxy (BGG), respectively. The BGG is located at $\left(\alpha_{\mathrm{J} 2000}, \delta_{\mathrm{J} 2000}\right)=\left(12^{\mathrm{h}} 06^{\mathrm{m}} 13.2,-08^{\circ} 47^{\prime} 45^{\prime \prime}\right)$, within the MUSTANG SZE residual region. Figure 14 shows an additional spiral galaxy at $\left(\alpha_{\mathrm{J} 2000}, \delta_{\mathrm{J} 2000}\right)=\left(12^{\mathrm{h}} 06^{\mathrm{m}} 13.3,-08^{\circ} 47^{\prime} 37^{\prime \prime}\right)$ that is coincident with an X-ray compact source and optically brighter than the BGG, but was originally assigned to the main cluster. Moreover, the Peak+Gap method (see Fadda et al. 1996; Biviano et al. 2013) used to assign member galaxies to the main cluster computes a $37 \%$ probability that this galaxy belongs with the $z \sim 0.42$ population instead, making it a likely member of the putative group.

We also use the Chandra data to provide a consistency check on the putative group's mass. Since the X-ray centroid of MACS J1206.2-0847 is shifted toward the east (see green " $X$ " in Figure 8), showing excess emission just south of the group's location on the sky, it is difficult to disambiguate the group's X-ray flux from that of MACS J1206.2-0847. Further, for masses consistent with the velocity dispersion mass estimate above, $R_{500}$ of the group lies entirely within that of MACS J1206.2-0847, so the X-ray background in this region is higher than it would be in a similar observation of an isolated group. Therefore, our estimate of the X-ray luminosity of the undetected group can only provide a weak upper limit of its mass.

Using an aperture corresponding to the $R_{500}$ of the optically identified group, the exposure-corrected $0.1-2.4 \mathrm{keV}$ Chandra image of MACS J1206.2-0847 yields an X-ray flux of $7.8_{-1.6}^{+0.9} \times 10^{-13} \mathrm{erg} \mathrm{s}^{-1} \mathrm{~cm}^{-2}$. This provides an upper limit on the soft $0.1-2.4 \mathrm{keV}$ X-ray luminosity of $L_{X}<4.3-6.1$ $\times 10^{44} \mathrm{erg} \mathrm{s}^{-1}$ for an infalling group masked by the $\mathrm{X}$-ray emission from MACS J1206.2-0847, which we have attempted to subtract from the flux estimate in this region. Using the Malmquist Bias corrected $L_{X}-M_{500}$ scaling relation of Pratt et al. (2009), which are consistent the $Y_{\mathrm{SZ}}-M$ and $Y_{\mathrm{SZ}}-L_{\mathrm{X}}$ scaling relations in $\mathrm{A} 10$, we place an upper limit of $M_{500}<(4.0-4.5) \times 10^{14} M_{\odot}$ for the region selected by the MUSTANG and VLT data.

Finally, we use a multi-halo NFW fitting procedure (see Medezinski et al. 2013) to derive weak-lensing mass estimates for the group by fitting two halos, namely the main cluster and the putative group, using the gravitational shear data presented in Umetsu et al. (2014; see also Umetsu et al. 2012). To do this, we construct a reduced-shear map on a regular grid of $42 \times 42$ independent cells, covering a $24 \times 24 \operatorname{arcmin}^{2}$ region centered on the BCG. We exclude from our analysis the $2 \times 2$ innermost cells lying in the supercritical (strong-lensing) regime.

We describe the primary cluster as an elliptical NFW (eNFW; see Umetsu et al. 2012; Medezinski et al. 2013) model with the centroid fixed at the BCG, thus specified with four parameters, namely, the halo mass $\left(M_{200}\right)$, concentration $\left(c_{200}\right)$, ellipticity $(e=1-b / a)$, and position angle of the major axis. We assume uniform priors for the halo mass, $M_{200}>0$, and the concentration, $3 \leqslant c_{200} \leqslant 6$, which is the range expected for CLASH X-ray selected clusters (see Meneghetti et al. 2014). For the group, we assume a spherical NFW model with the
Table 7

Group Mass Estimates

\begin{tabular}{lc}
\hline \hline Method & $M_{500}$ \\
& $\left(10^{14} M_{\odot}\right)$ \\
\hline MUSTANG SZE & $>0.13$ \\
X-ray & $<4.5$ \\
$\sigma_{\mathrm{V}}-M(\mathrm{VLT})$ & $1.4 \pm 0.9$ \\
eNFW+NFW & $2.2 \pm 1.2$ \\
\hline
\end{tabular}

Note. Summary of the mass constraints and estimates derived from the SZE, X-ray, VLT, and weak-lensing data.

centroid fixed at $\left(\alpha_{\mathrm{J} 2000}, \delta_{\mathrm{J} 2000}\right)=\left(12^{\mathrm{h}} 06^{\mathrm{m}} 13.3,-08^{\circ} 47^{\prime} 37^{\prime \prime}\right)$, and the redshift at $z=0.423$. We assume a flat prior for the group halo mass, $M_{200}<7.5 \times 10^{14} M_{\odot}$, corresponding to the X-ray-derived upper limit of $M_{500} \sim 4.5 \times 10^{14} M_{\odot}$ (Table 7), and adopt the $c-M$ relation from Bhattacharya et al. (2013). We marginalize over the source redshift uncertainty reported in Table 3 of Umetsu et al. (2014).

The resulting two halo model is shown in Figure 13 (blue contours). From the simultaneous two-component (eNFW $+\mathrm{NFW})$ fit to the two-dimensional reduced shear data, we determine a group mass of $M_{200}=3.6 \pm 2.0 \times 10^{14} M_{\odot}$, or $M_{500}=2.2 \pm 1.2 \times 10^{14} M_{\odot}$. In this two-halo fit, we find the best value of the primary cluster mass to be $M_{200}=9.4 \pm$ $3.1 \times 10^{14} M_{\odot}$, or $M_{500}=5.6 \pm 1.9 \times 10^{14} M_{\odot}$. We note that these results are sensitive to the assumed priors as the weaklensing data do not resolve the group.

The X-ray and SZE measurements place constraints on the group mass that, while not stringent, are consistent with the mass estimates from the velocity dispersion and the multi-halo eNFW+NFW fitting (see Table 7).

\subsubsection{Extended Radio Emission}

GMRT observations at $610 \mathrm{MHz}$ reveal extended diffuse radio emission west of the central AGN (Figures 8 and 9), which is likely an AGN-driven plasma bubble or jet. However, such lobes are generally produced as symmetric pairs powered by the central black hole. The middle panel of Figure 9 shows that, after point source subtraction to account for the AGN, there is an excess amount of pressure east of the diffuse radio emission. This excess is associated with the core of the best-fit gNFW model (Table 3) that describes the MUSTANG and Bolocam data (yellow " $X$ " in Figure 8). We note this model has a steeper inner profile than the median A10 universal pressure profile. We posit that this positional offset leads to suppression of the would-be eastern radio lobe, while the western lobe appears be expanding asymmetrically away from this higher pressure region.

The offset between the pressure profile and the AGN/BCG seems to suggest the ICM is sloshing subsonically, as sloshing should not produce strong pressure discontinuities (ZuHone et al. 2013). This scenario is supported by the $\sim 7^{\prime \prime}$ offset between the centroid of the X-ray emitting gas and the BCG location (Figure 8), along with the general E-W elongation of both the surface mass distribution seen in strong-lensing and the X-ray surface brightness.

The strong-lensing data in Zitrin et al. (2012) (reproduced in Figure 8 ) reveal a massive component $\sim 40^{\prime \prime}$ to the east of the cluster core. If this subcluster has passed in front of or behind the main cluster's core, it may have induced E-W sloshing that 
has redirected one or both jets away from the line of sight. In this case, the radio emission observed could be a superposition of both lobes, or one lobe could be masked by the bright AGN emission. Sloshing also allows for the possibility that a detached, aged lobe or bubble was compressed adiabatically, re-accelerating its relativistic electrons to emit in the radio (e.g., Clarke et al. 2013). Deeper multi-band radio data are required to measure the spectral index of the diffuse emission to distinguish the possibilities. In addition, higher resolution radio data are necessary to understand the nature of the western radio feature and its interaction with the surrounding ICM and connection to, or detachment from, the AGN.

In the sloshing scenario outlined above, the MUSTANG SZE residual substructure (right panel of Figure 9) is most plausibly an interloping foreground structure associated with the group discussed in Section 5.2.2.

\section{CONCLUSION}

We have presented high-resolution images of the SZE from MUSTANG observations of MACS J0647.7+7015 and MACS J1206.2-0847. We compare the MUSTANG measurements to cluster profiles derived from fits to lower resolution Bolocam SZE data and find that in general a steeper core profile is called for compared to the universal pressure profile from A10. We caution that the serial-fitting approach in this work inherently treats the fit to the Bolocam data as an additional prior on the final fit to the MUSTANG data, and thus does not provide the statistically accurate error bars one can obtain from a joint fitting procedure. We thus refer the reader to Romero et al. (2015) for a more comprehensive joint analysis of MUSTANG + Bolocam data.

We use archival Chandra data to generate pseudo-SZE models for both MACS J0647.7+7015 and MACS J1206.2-0847, which we normalize based on the integrated flux within a $1^{\prime}$ radius from the Bolocam observations. We find that the Bolocam SZE profile in the core is shallower than the pseudo-SZE, which we attribute to smoothing by the Bolocam PSF on the scales shown in these maps.

In MACS J0647.7+7015 the MUSTANG SZE decrement closely follows the shape and flux expected from the X-ray pseudo-SZE map. The MUSTANG and Bolocam data are well described by a gNFW model with $\gamma=0.9$.

MUSTANG detects the central AGN in MACS J1206.2-0847 in addition to an excess of SZE emission to the NE. We compare the MUSTANG data to models derived from Bolocam and find that a gNFW with $\gamma=0.7$ best describes the data. After accounting for the point source and primary ICM distribution, MUSTANG measures a $\sim 3 \sigma$ residual decrement to the NE. Using spectroscopic redshift measurements, we carry out a kinematic analysis of the galaxies surrounding the main cluster and find evidence for a 13 member group at $z \sim 0.42$. From the X-ray and SZE data we derive upper and lower bounds, respectively, for the mass of this group. We carry out a multi-halo fit to constrain a weaklensing mass estimate for the group and find good agreement with the mass derived from the VLT data.

Observations with the GMRT at $610 \mathrm{MHz}$ reveal extended radio emission west of the central AGN. We suggest that this emission is an AGN-driven plasma bubble or jet. While deeper multi-wavelength and higher resolution data are required to characterize this feature, the asymmetric morphology of the proposed jet could be explained by sloshing of the ICM or an infalling group to the NE.

We thank the anonymous referee for the useful comments that helped improve this manuscript. The National Radio Astronomy Observatory is a facility of the National Science Foundation operated under cooperative agreement by Associated Universities, Inc. The GBT+MUSTANG observations presented here were obtained with telescope time allocated under NRAO proposal IDs AGBT11A009, and AGBT11B001. Additional funding was provided by NSF/AST-1309032. The Bolocam observations presented here were obtained operating from the Caltech Submillimeter Observatory, which, when the data used in this analysis were taken, was operated by the California Institute of Technology under cooperative agreement with the National Science Foundation. Bolocam was constructed and commissioned using funds from NSF/AST9618798, NSF/AST-0098737, NSF/AST-9980846, NSF/ AST-0229008, and NSF/AST-0206158. Bolocam observations were partially supported by the Gordon and Betty Moore Foundation, the Jet Propulsion Laboratory Research and Technology Development Program, as well as the National Science Council of Taiwan grant NSC100-2112-M-001-008MY3. The MACS J1206.2-0847 spectroscopic data were based on the ESO VLT Large Programme (prog. ID 186.A-0798, PI: P. Rosati). Basic research in radio astronomy at the Naval Research Laboratory is supported by 6.1 Base funding. The late night assistance of the GBT operators Greg Monk, Donna Stricklin, Barry Sharp and Dave Rose was much appreciated during the observations. Much of the work presented here was supported by NSF grant AST-0607654. Support for TM was provided by NASA through the Einstein Fellowship Program, grant PF0-110077, and through a National Research Council Research Associateship Award at the U.S. Naval Research Laboratory. Support for P.K. and A.Y. was provided by the NRAO Student Observing Support (SOS) and NASA Postdoctoral Fellowship programs. J.S. was partially supported by a Norris Foundation CCAT Postdoctoral Program Fellowship and by NSF/AST-1313447.

\section{REFERENCES}

Agudo, I., Thum, C., Wiesemeyer, H., et al. 2012, A\&A, 541, A111 Annunziatella, M., Biviano, A., Mercurio, A., et al. 2014, A\&A, 571, A80 Arnaud, M., Pratt, G. W., Piffaretti, R., et al. 2010, A\&A, 517, A92 (A10) Bhattacharya, S., Habib, S., Heitmann, K., \& Vikhlinin, A. 2013, ApJ, 766, 32 Birkinshaw, M. 1999, PhR, 310, 97

Biviano, A., Rosati, P., Balestra, I., et al. 2013, A\&A, 558, A1 Carlstrom, J. E., Holder, G. P., \& Reese, E. D. 2002, ARA\&A, 40, 643 Cassano, R., Brunetti, G., Norris, R. P., et al. 2012, A\&A, 548, A100 Cavagnolo, K. W., Donahue, M., Voit, G. M., \& Sun, M. 2009, ApJS, 182, 12 Clarke, T. E., Randall, S. W., Sarazin, C. L., Blanton, E. L., \& Giacintucci, S. 2013, ApJ, 772, 84

Czakon, N. G., Sayers, J., Mantz, A., et al. 2015, ApJ, 806, 18

den Hartog, R., \& Katgert, P. 1996, MNRAS, 279, 349

Dicker, S. R., Korngut, P. M., Mason, B. S., et al. 2008, Proc. SPIE, 7020, 5

Ebeling, H., Barrett, E., Donovan, D., et al. 2007, ApJL, 661, L33

Ebeling, H., Edge, A. C., \& Henry, J. P. 2001, ApJ, 553, 668

Ebeling, H., Ma, C. J., Kneib, J.-P., et al. 2009, MNRAS, 395, 1213

Fadda, D., Girardi, M., Giuricin, G., Mardirossian, F., \& Mezzetti, M. 1996, ApJ, 473, 670

Ferrari, C., Intema, H. T., Orrù, E., et al. 2011, A\&A, 534, L12

Gilmour, R., Best, P., \& Almaini, O. 2009, MNRAS, 392, 1509

Haig, D. J., Ade, P. A. R., Aguirre, J. E., et al. 2004, Proc. SPIE, 5498, 78

Hung, C.-L., \& Ebeling, H. 2012, MNRAS, 421, 3229

Itoh, N., Kohyama, Y., \& Nozawa, S. 1998, ApJ, 502, 7

Itoh, N., \& Nozawa, S. 2004, A\&A, 417, 827 
Jewell, P. R., \& Prestage, R. M. 2004, Proc. SPIE, 5489, 312

Kitayama, T., Komatsu, E., Ota, N., et al. 2004, PASJ, 56, 17

Komatsu, E., Matsuo, H., Kitayama, T., et al. 2001, PASJ, 53, 57

Korngut, P. M., Dicker, S. R., Reese, E. D., et al. 2011, ApJ, 734, 10

Mann, A. W., \& Ebeling, H. 2012, MNRAS, 420, 2120

Mantz, A., Allen, S. W., Ebeling, H., Rapetti, D., \& Drlica-Wagner, A. 2010, MNRAS, 406, 1773

Mason, B. S., Dicker, S. R., Korngut, P. M., et al. 2010, ApJ, 716, 739

Medezinski, E., Umetsu, K., Nonino, M., et al. 2013, ApJ, 777, 43

Meneghetti, M., Rasia, E., Vega, J., et al. 2014, ApJ, 797, 34

Mroczkowski, T., Bonamente, M., Carlstrom, J. E., et al. 2009, ApJ, 694, 1034

Mroczkowski, T., Dicker, S., Sayers, J., et al. 2012, ApJ, 761, 47

Munari, E., Biviano, A., Borgani, S., Murante, G., \& Fabjan, D. 2013, MNRAS, 430, 2638

Nagai, D., Kravtsov, A. V., \& Vikhlinin, A. 2007, ApJ, 668, 1

Nikolic, B., Prestage, R. M., Balser, D. S., Chandler, C. J., \& Hills, R. E. 2007, A\&A, 465, 685

Plagge, T., Benson, B. A., Ade, P. A. R., et al. 2010, ApJ, 716, 1118

Planck Collaboration, Ade, P. A. R., Aghanim, N., et al. 2013a, A\&A, 550, A131

Planck Collaboration, Ade, P. A. R., Aghanim, N., et al. 2013b, A\&A, 550, A132

Planck Collaboration, Ade, P. A. R., Aghanim, N., et al. 2014, A\&A, 571, A16
Pointecouteau, E., Giard, M., Benoit, A., et al. 1999, ApJL, 519, L115

Postman, M., Coe, D., Benítez, N., et al. 2012, ApJS, 199, 25

Pratt, G. W., Croston, J. H., Arnaud, M., \& Böhringer, H. 2009, A\&A, 498, 361

Press, W. H., Teukolsky, S. A., Vetterling, W. T., \& Flannery, B. P. 1992, Numerical Recipes in C. The Art of Scientific Computing (2nd ed. Cambridge: Cambridge Univ. Press)

Reese, E. D., Kawahara, H., Kitayama, T., et al. 2010, ApJ, 721, 653

Romero, C., Mason, B. S., Sayers, J., et al. 2015, ApJ, 807, 121

Sayers, J., Czakon, N. G., Mantz, A., et al. 2013, ApJ, 768, 177 (S13)

Sayers, J., Golwala, S. R., Ameglio, S., \& Pierpaoli, E. 2011, ApJ, 728, 39

Sunyaev, R. A., \& Zel'dovich, Y. B. 1972, CoASP, 4, 173

Umetsu, K., Medezinski, E., Nonino, M., et al. 2012, ApJ, 755, 56

Umetsu, K., Medezinski, E., Nonino, M., et al. 2014, ApJ, 795, 163

van Weeren, R. J., Intema, H. T., Röttgering, H. J. A., Brüggen, M., \& Hoeft, M. 2011, MmSAI, 82, 569

Vollmer, B., Gassmann, B., Derrière, S., et al. 2010, A\&A, 511, A53

Weiland, J. L., Odegard, N., Hill, R. S., et al. 2011, ApJS, 192, 19

Zitrin, A., Broadhurst, T., Barkana, R., Rephaeli, Y., \& Benítez, N. 2011 , MNRAS, 410, 1939

Zitrin, A., Rosati, P., Nonino, M., et al. 2012, ApJ, 749, 97

ZuHone, J. A., Markevitch, M., Ruszkowski, M., \& Lee, D. 2013, ApJ, 762,69 